\title{
An X-ray activity cycle on the young solar-like star $\epsilon$ Eridani
}

\author{
M. Coffaro ${ }^{1}$, B. Stelzer ${ }^{1,2}$, S. Orlando ${ }^{2}$, J. Hall ${ }^{3}$, T.S. Metcalfe ${ }^{4}$, U. Wolter ${ }^{5}$, M. Mittag ${ }^{5}$, J. Sanz-Forcada ${ }^{6}$, P.C. \\ Schneider ${ }^{5}$, and L. Ducci ${ }^{1}$ \\ ${ }^{1}$ Institut für Astronomie und Astrophysik Tübingen (IAAT), Eberhard-Karls Universität Tübingen, Sand 1, D-72076, Germany e- \\ mail: coffaro@astro.uni-tuebingen.de \\ 2 INAF - Osservatorio Astronomico di Palermo, Piazza del Parlamento 1, I-90134 Palermo, Italy \\ 3 Lowell Observatory, 1400 West Mars Hill Road, Flagstaff, AZ 86001 \\ 4 Space Science Institute, 4765 Walnut Street, Suite B, Boulder, CO 80301, USA \\ 5 Hamburger Sternwarte, Universität Hamburg, Gojenbergsweg 112, 21029, Hamburg, Germany \\ ${ }^{6}$ Departamento de Astrofísica, Centro de Astrobiología (CSIC-INTA), ESAC Campus, Camino bajo del Castillo s/n, E-28692 Vil- \\ lanueva de la Cañada, Madrid, Spain.
}

Received 8 August 2019 / Accepted 20 February 2020

\section{ABSTRACT}

\begin{abstract}
Chromospheric Ca II activity cycles are frequently found in late-type stars, but there have been no systematic programs to search for their coronal X-ray counterparts. The typical time scale of Ca II activity cycles goes from years to decades. Therefore, long-lasting missions are needed to detect the coronal counterparts. XMM-Newton has so far detected X-ray cycles in five stars. A particularly intriguing question is at what age (and at what activity level) X-ray cycles set in. To this end, in 2015 we started the X-ray monitoring of the young solar-like star $\epsilon$ Eridani, observed previously twice in 2003 and in early 2015 by XMM-Newton. With an age of 440 Myr, it is one of the youngest solar-like stars with a known chromospheric Ca II cycle. We collected the most recent Mount Wilson S-index data available for $\epsilon$ Eridani, starting from 2002, including previously unpublished data. We found that the Ca II cycle lasts $2.92 \pm 0.02$ $\mathrm{yr}$, in agreement with past results. From the long-term XMM-Newton lightcurve, we find clear and systematic X-ray variability of our target, consistent with the chromospheric Ca II cycle. The average X-ray luminosity results to be $2 \times 10^{28} \mathrm{erg} / \mathrm{s}$, with an amplitude that is only a factor 2 throughout the cycle. We apply a new method to describe the evolution of the coronal emission measure distribution of $\epsilon$ Eridani in terms of solar magnetic structures: active regions, cores of active regions and flares covering the stellar surface at varying filling fractions. Combinations of these three types of magnetic structures can describe the observed X-ray emission measure of $\epsilon$ Eridani only if the solar flare emission measure distribution is restricted to events in the decay phase. The interpretation is that flares in the corona of $\epsilon$ Eridani last longer than their solar counterparts. We ascribe this to the lower metallicity of $\epsilon$ Eridani. Our analysis revealed also that the X-ray cycle of $\epsilon$ Eridani is strongly dominated by cores of active regions. The coverage fraction of cores throughout the cycle changes by the same factor as the X-ray luminosity. The maxima of the cycle are characterized by a high percentage of covering fraction of the flares, consistent with the fact that flaring events are seen in the corresponding short-term X-ray lightcurves predominately at the cycle maxima. The high X-ray emission throughout the cycle of $\epsilon$ Eridani is thus explained by the high percentage of magnetic structures on its surface.
\end{abstract}

Key words. X-ray: stars - Stars: solar-type - Stars: activity - Stars: coronae - Stars: individual: $\epsilon$ Eri

\section{Introduction}

Stellar dynamos maintain the magnetic field of late-type stars on long-timescales through a periodic field reversal. The dynamo cycle comprises a phase in which the interior field rises to the surface where it forms magnetic loop structures. As yet poorly determined, magnetic heating processes produce a positive temperature gradient above the photosphere, and ensuing characteristic emission from plasma at $T \sim 10000 \mathrm{~K}$ to few $10^{6} \mathrm{~K}$. As the surface coverage with these high-activity regions changes throughout the dynamo cycle, an activity cycle of the same length is associated with the magnetic field cycle. Observations of such activity cycles can, thus, be used as proxies for the stellar dynamo.

In the chromosphere, the monitoring of $\mathrm{H}$ and $\mathrm{K}$ emission lines of $\mathrm{Ca}$ II is the most widely used indicator for activity cycles. In a dedicated monitoring program at the Mt. Wilson telescope (Wilson 1968, 1978), the ubiquitous existence of cyclic stellar activity in cool stars was revealed: about $60 \%$ of the mainsequence stars with spectral types $\mathrm{F}$ and $\mathrm{M}$ present variability of the Ca II H\&K Mount Wilson S-index, $S_{\text {Mwo }}$, (Baliunas et al. 1995), showing periodicities from $2 \mathrm{yr}$ up to $20 \mathrm{yr}$. Finding the $\mathrm{X}$-ray counterpart of activity cycles, i.e. their manifestation in the stellar corona, is still challenging. Due to the typical cycle length of years to decades, long-term X-ray monitoring campaigns of activity cycles are unfeasible for a significant sample. Such studies require a long-lived X-ray mission, as problems of cross-calibration can arise when different telescopes are used, because the data would have different wavelength coverage and responses.

Up to date, XMM-Newton has detected X-ray activity cycles in five stars ${ }^{1}$. Four of these stars are part of stellar systems: 61

\footnotetext{
1 Among the stars observed multiple times with XMM-Newton, other two stars show variability in the X-ray waveband, compatible with the chromospheric cycles observed during the Mount Wilson project. These stars are $\tau$ Boötis (Mittag et al. 2017), with a cycle period of $\sim 4$ months, the shortest period observed, and the third companion of the
} 
Cyg A (Hempelmann et al. 2006; Robrade et al. 2012), $\alpha$ Cen A and $\alpha$ Cen B (Robrade et al. 2012; Wargelin et al. 2016) and HD 81809 (Favata et al. 2008; Orlando et al. 2017). They are old stars with ages of several Gyr and they show long X-ray cycle periods, from $8 \mathrm{yr}$ to $\sim 19 \mathrm{yr}$. Favata et al. (2008) and Orlando et al. (2017) have hypothesized that the stellar X-ray activity of HD 81809 comes from the primary component of the binary system. This statement was questioned in the literature (Radick et al. 2018), but the main obstacle in constraining such systems comes from the geometrical configuration of the system that is not spatially resolved (Egeland 2018).

The fourth star monitored by XMM-Newton, $\iota$ Horologii, has different characteristics from the others: it is relatively young with an age of $\sim 600 \mathrm{Myr}$ and a cycle period of $1.6 \mathrm{yr}$, the shortest X-ray cycle measured until now (Sanz-Forcada et al. 2013). The detection of an X-ray cycle in $\iota$ Hor has shown that coronal cycles can be also found in young stars. This triggers the question at which age and at which activity level X-ray cycles set in. To this end, we need well-selected targets such as $\iota$ Hor, i.e. young and active solar-like stars with short chromospheric cycle period, enabling an X-ray detection in reasonable time-span.

$\epsilon$ Eridani ( $\epsilon$ Eri; HD 22049) is a young roughly solar-type dwarf star with spectral type K2V. It is at a distance of $3.2 \mathrm{pc}$ (van Leeuwen 2007), has an age of $440 \mathrm{Myr}^{2}$ and a radius of $0.74 R_{\odot}$ (Barnes 2007; Di Folco et al. 2004). It hosts two Jupiterlike planets with a semimajor axis of $3.4 \mathrm{AU}$ and $40 \mathrm{AU}$ respectively (Hatzes et al. 2000; Quillen \& Thorndike 2002). $\epsilon$ Eri was part of the Mt. Wilson project, and the monitoring of its chromospheric emission started in 1968. These data were first published by Gray \& Baliunas (1995), finding a cycle period of approximately 5 yr. Later Metcalfe et al. (2013) combined the Mount Wilson data with more recent observations carried out at other observatories. They found two periodicities in the longterm lightcurve of the S-index: $2.95 \pm 0.03 \mathrm{yr}$ and $12.7 \pm 0.3$ yr.

The clear evidence for a short chromospheric cycle together with its youth, have led us to start an XMM-Newton X-ray monitoring program of $\epsilon$ Eri. Here we report the first detection of a $\sim 3$ yr X-ray cycle.

In Sect. 2 we present the most recent Ca II $S_{\text {MwO-index data }}$ and our X-ray monitoring campaign of $\epsilon$ Eri, together with the description of the data reduction. In Sect. 3 we present a novel method in which we describe the X-ray emission and the evolution of the coronal cycle of $\epsilon$ Eri in terms of varying solar-like magnetic structures. It consists in comparing the emission measure distributions (EMDs) of the magnetic structures observed on the Sun to that of $\epsilon$ Eri. Favata et al. (2008) and Orlando et al. (2017) laid the foundations for this technique in application to HD 81809. Here, the high-quality spectra of $\epsilon$ Eri allow us here a much more detailed study in which we also refine the method. In Sect. 4 and Sect. 5 we discuss our results and we give our conclusions.

\section{Observations and data analysis}

\subsection{Ca II H\&K data}

As support of our search for an X-ray activity cycle, we collected

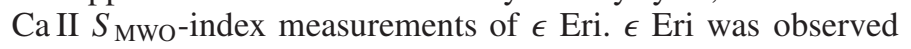

stellar system $\alpha$ Cen, Proxima Cen, with an evidence of an activity cycle recently found (Wargelin et al. 2016).

2 In the literature the age of $\epsilon$ Eri is estimated to be between $200 \mathrm{Myr}$ and $930 \mathrm{Myr}$ (Fuhrmann 2004; Song et al. 2000). We adopt here the age of 440 Myr found by Barnes (2007) through gyro-chronology. within the Mount Wilson project, from the late 60s to early 90s. After the Mount Wilson project the monitoring of the Ca II H\&K lines continued at other observatories. The data we take into account in this article for $\epsilon$ Eri were obtained from 2002 to late 2018 such as to cover the full time-span of the existing XMMNewton observations of $\epsilon$ Eri.

Our data set comes from different instruments: Solar-Stellar Spectrograph (SSS) at Lowell Observatory in Arizona, SMARTS (Small and Moderate Aperture Research Telescope System) $1.5 \mathrm{~m}$ telescope in Chile and TIGRE telescope at La Luz Observatory in Mexico. These data are described in the subsequent sections. The chromospheric variability of $\epsilon$ Eri was also observed by HIRES at the Keck Observatory in Hawaii, and the data were published by Isaacson \& Fischer (2010). All the $S_{\mathrm{MWO}}$-index measurements are shown in Fig. 1.

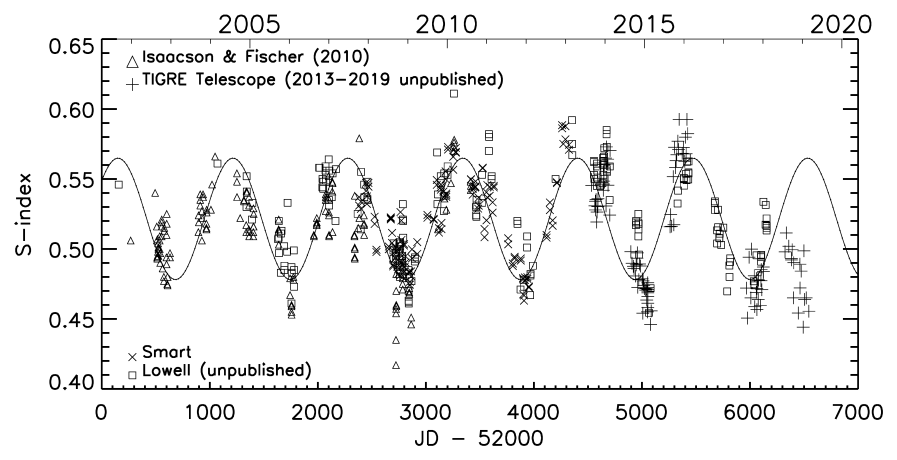

Fig. 1: Ca II Mount Wilson S-index of $\epsilon$ Eri. The data set covers a period from 2002 to late 2018 and it comes from four different instruments: SMARTS $1.5 \mathrm{~m}$ telescope (cross-symbols), SSS at Lowell Observatory (squared-symbols), HIRES at the Keck Observatory (triangle-symbols) and TIGRE telescope at La Luz Observatory (plus-symbols). The solid line is the sinusoidal function representing the peak with the highest power in the periodogram (see Sect. 2.1.4).

\subsubsection{Lowell Observatory}

The Lowell Observatory Solar-Stellar Spectrograph (SSS) records the entire spectrum surrounding Ca II H\&K from 386 to $401 \mathrm{~nm}$. Regular observations of $\sim 100$ stars, including $\epsilon$ Eri, have been ongoing since 1994. We collected for $\epsilon$ Eri a total of 260 observations from 1994 to the late 2018, and we considered for our analysis the data starting from 2002. We performed the SSS data reduction using a set of IDL routines, employing the usual sequence of spectroscopic data reduction steps. To obtain the S-index, we first placed each SSS H\&K spectrum on an absolute intensity scale using pseudo-continuum reference points at 312 and $400 \mathrm{~nm}$, as described by Hall \& Lockwood (1995). We measured the total residual emission in $0.1 \mathrm{~nm}$ rectangular bandpasses centered on the $\mathrm{H}$ and $\mathrm{K}$ line cores, and then convert this raw $\mathrm{HK}$ index, $F_{H K}$, to flux and hence to S-index, following the prescription of Rutten (1984) with modifications presented by Hall et al. (2007). The resulting calibration is quite satisfactory; for the full 25-year SSS time series of 23 flat-activity stars for which we also have long-term records from Mount Wilson, a linear regression yields $S_{M W O}=0.976 S_{S S S}+0.0044$ (Hall et al. 2019 , in prep). The $S S S$ grand mean $S_{M W O}$-index we obtained for $\epsilon$ Eri is 0.528 . 


\subsubsection{SMARTS}

Observations from the SMARTS southern HK project (Metcalfe et al. 2009) include 148 low-resolution $(R \sim 2500)$ spectra obtained on 74 distinct epochs between August 2007 and January 2013 using the $R C$ Spec instrument on the $1.5-\mathrm{m}$ telescope at Cerro Tololo Interamerican Observatory. Bias and flat-field corrections were applied to the $60 \mathrm{~s}$ integrations, and the wavelength was calibrated using standard IRAF routines. $S_{M W O}$ values were extracted from the reduced spectra following Duncan et al. (1991), placing the instrumental measurements onto the Mount Wilson scale using contemporaneous observations from the SSS instrument. The mean of the $S_{M W O}$ values is 0.513 .

\subsubsection{TIGRE telescope}

The TIGRE spectra were reduced using version 3 of the TIGRE/HEROS pipeline (Mittag et al. 2018), based on the REDUCE package (Piskunov \& Valenti 2002). The pipeline follows the usual steps of reducing echelle CCD frames; it automatically computes line core indices of activity-sensitive lines, including the combined Ca II H\&K lines, $S_{\text {TIGRE. The compu- }}$ tation of the $S_{\text {TIGRE }}$ indices is analogous to the computation of the $S_{\mathrm{MWO}}$-index and they were converted using the equation $S_{M W O}=0.0360+20.02 \cdot S_{\text {TIGRE }}$, according to Mittag et al. (2016) (see Fig. 1 of that paper). The TIGRE data set covers a temporal range from 2013 to the end of 2018, comprising in total 86 spectra, and the mean of these measurements results $S_{M W O}=0.514$.

\subsubsection{Chromospheric cycle of $\epsilon$ Eri}

The $S_{\text {MWO-index obtained from the three instruments considered }}$ in this work are in good agreement with each other. The shortterm scatter of the $S_{\text {MWO-index seen in Fig. } 1 \text { is potentially due }}$ to the rotational modulation of $\epsilon \mathrm{Eri}^{3}$.

Metcalfe et al. (2013) calculated the cycle period based on data covering the years from 1992 to 2013. Our time range covers more recent years, until the end of 2018 . We consider $S_{\text {MwO- }^{-}}$ index measurements starting in 2002, covering thus the time range of the XMM-Newton observations, and we performed the period search on this dataset.

The Lomb-Scargle periodogram was calculated using the software GLS (Generalized Lomb-Scargle Periodogram), implemented by Zechmeister \& Kürster (2009). We found a period of 1067.13 days. The Lomb-Scargle periodogram is shown in Fig. 2, with the window function in the bottom panel. The error on the cycle period was found through Monte-Carlo simulations. We simulated 10000 data sets of the Ca II $S_{\text {MWO-index }}$ measurements. Each data point was randomly drawn from a normal distribution within the observed standard deviation around the measured $S_{\mathrm{MWO}}$-index. We performed a Lomb-Scargle analysis for each simulated data set, obtaining 10000 values of the period; the standard deviation of these values was then considered as the error of the cycle period. To summarize, we found a period of $2.92 \pm 0.02 \mathrm{yr}$. This value, and its amplitude resulting from the GLS analysis, were used to plot the sinusoidal function in Fig. 1.

\footnotetext{
3 A study of rotational effects is outside the scope of this work. See e.g. Jeffers et al. (2014) for measurements of rotational modulation of various emission lines of $\epsilon$ Eri.
}

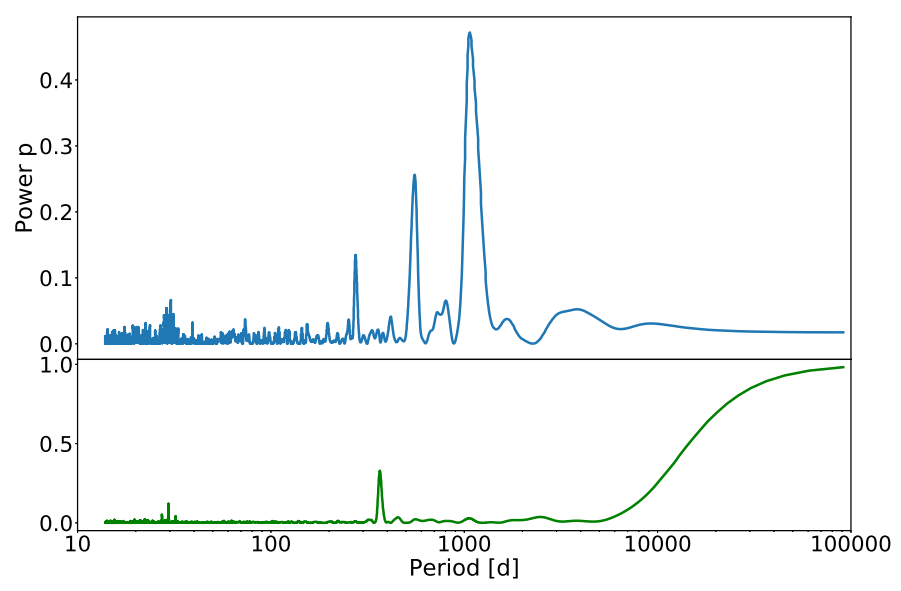

Fig. 2: Lomb-Scargle periodogram of the Ca II S-index data. The bottom panel represents the window function. For the data set that covers in total 15 years, the most significant peak is found at $2.92 \mathrm{yr}$.

Our result is consistent with the value of $2.95 \pm 0.03 \mathrm{yr}$ found by Metcalfe et al. (2013). However, we did not find the second periodicity at $12.7 \mathrm{yr}$. That signal was rooted in the broad minimum from 1984 to 1996, while our data set covers a more recent time range. During the years relevant for our work, i.e. the time covered by XMM-Newton, cleary the short cycle was dominant. Moreover, the most recent maximum expected around 2019 was not seen, suggesting another change in cycle behavior.

\subsection{X-ray data}

Our X-ray monitoring campaign of $\epsilon$ Eri started in July 2015. The observations were carried out with the XMM-Newton satellite. In this work we present seven snapshots with an exposure time varying between 6 and $20 \mathrm{ks}$.

TABLE 1: Observing log of XMM-Newton data for $\epsilon$ Eri.

\begin{tabular}{cccc}
\hline \hline Date & Rev. & $\begin{array}{c}\text { Science Mode } \\
(\text { EPIC/pn })\end{array}$ & $\begin{array}{c}\text { Exposure Time } \\
(\mathrm{ks})\end{array}$ \\
\hline $2003-01-19$ & 0570 & Full Window & 11.5 \\
$2015-02-02$ & 2775 & Large Window & 16.7 \\
$2015-07-19$ & 2858 & Small Window & 6.5 \\
$2016-01-31$ & 2957 & Small Window & 7.7 \\
$2016-07-19$ & 3042 & Small Window & 8.9 \\
$2017-01-16$ & 3133 & Small Window & 6.0 \\
$2017-08-26$ & 3244 & Small Window & 8.8 \\
$2018-01-16$ & 3316 & Small Window & 6.5 \\
$2018-07-20$ & 3408 & Small Window & 19.6 \\
\hline
\end{tabular}

XMM-Newton allows to monitor the target using two X-ray instruments simultaneously: EPIC (European Imaging Photon Camera) pn detector covers the energy band $0.15-15 \mathrm{keV}$ and EPIC MOS detector 0.2 - $10 \mathrm{keV}$; RGS (Reflection Grating Spectrometer) produces high-resolution spectra. Our observations were conducted in EPIC Small Window mode. Previous to our campaign, $\epsilon$ Eri had been observed twice by XMM-Newton in Full and Large Frame mode. These two observations are also considered in our analysis. The observing log is given in Table 1. We focus on the analysis of the data from the EPIC/pn instrument: adding the EPIC/MOS would considerably complicate the subsequent analysis (see Sect. 3), without adding relevant infor- 
TABLE 2: Best-fit spectral parameters of each XMM-Newton EPIC/pn observation of $\epsilon$ Eri.

\begin{tabular}{|c|c|c|c|c|c|c|c|c|c|c|c|}
\hline Obs No. & "Rev & $\begin{array}{l}k T_{1} \\
k e V\end{array}$ & $\overline{c k T_{2}}$ & $\overline{k k T_{3}}$ & $\begin{array}{c}\log E M_{1} \\
\mathrm{~cm}^{-3}\end{array}$ & $\begin{array}{c}\log E M_{2} \\
\mathrm{~cm}^{-3}\end{array}$ & $\begin{array}{c}\log E M_{3} \\
\mathrm{~cm}^{-3}\end{array}$ & $\begin{array}{c}\text { Flux } \\
{[0.2-2 \mathrm{keV}]} \\
10^{-11} \mathrm{erg} \mathrm{cm}^{-2} \mathrm{~s}^{-1}\end{array}$ & $\begin{array}{c}L_{\mathrm{X}} \\
{[0.2-2 \mathrm{keV}]} \\
10^{28} \text { erg s}^{-1}\end{array}$ & $\begin{array}{l}T_{\mathrm{av}} \\
k e V\end{array}$ & $\overline{\overline{\bar{\chi}^{2}}}$ \\
\hline 1 & 0507 & $0.15 \pm 0.01$ & $0.33 \pm 0.02$ & $0.75 \pm 0.02$ & $50.70 \pm 0.06$ & $50.83 \pm 0.04$ & $50.55 \pm 0.04$ & $1.29 \pm 0.01$ & $1.58 \pm 0.01$ & $0.37 \pm 0.02$ & 1.02 \\
\hline 2 & 2775 & $0.15 \pm 0.02$ & $0.33 \pm 0.03$ & $0.78 \pm 0.05$ & $50.65 \pm 0.09$ & $50.81 \pm 0.06$ & $50.39 \pm 0.08$ & $1.10 \pm 0.03$ & $1.35 \pm 0.04$ & $0.35 \pm 0.03$ & 0.81 \\
\hline 3 & 2858 & $0.15 \pm 0.02$ & $0.34 \pm 0.02$ & $0.77 \pm 0.04$ & $50.81 \pm 0.05$ & $51.01 \pm 0.04$ & $50.60 \pm 0.05$ & $1.74 \pm 0.02$ & $2.13 \pm 0.02$ & $0.37 \pm 0.02$ & 1.08 \\
\hline 4 & 2957 & $0.16 \pm 0.02$ & $0.33 \pm 0.02$ & $0.74 \pm 0.04$ & $50.78 \pm 0.08$ & $51.00 \pm 0.04$ & $50.57 \pm 0.06$ & $1.69 \pm 0.02$ & $2.06 \pm 0.02$ & $0.35 \pm 0.02$ & 1.00 \\
\hline 5 & 3042 & $0.19 \pm 0.02$ & $0.36 \pm 0.03$ & $0.79 \pm 0.03$ & $50.85 \pm 0.08$ & $50.87 \pm 0.10$ & $50.83 \pm 0.04$ & $1.92 \pm 0.02$ & $2.35 \pm 0.02$ & $0.44 \pm 0.03$ & 1.00 \\
\hline 6 & 3133 & $0.19 \pm 0.03$ & $0.33 \pm 0.04$ & $0.73 \pm 0.03$ & $50.73 \pm 0.12$ & $50.83 \pm 0.13$ & $50.64 \pm 0.05$ & $1.47 \pm 0.02$ & $1.80 \pm 0.02$ & $0.39 \pm 0.05$ & 0.95 \\
\hline 7 & 3244 & $0.15 \pm 0.02$ & $0.32 \pm 0.02$ & $0.72 \pm 0.04$ & $50.68 \pm 0.08$ & $50.95 \pm 0.04$ & $50.47 \pm 0.06$ & $1.40 \pm 0.01$ & $1.72 \pm 0.02$ & $0.34 \pm 0.02$ & 1.17 \\
\hline 8 & 3316 & $0.21 \pm 0.02$ & $0.36 \pm 0.08$ & $0.78 \pm 0.03$ & $50.89 \pm 0.17$ & $50.85 \pm 0.20$ & $50.83 \pm 0.05$ & $1.97 \pm 0.02$ & $2.41 \pm 0.02$ & $0.43 \pm 0.05$ & 0.90 \\
\hline 9 & 3408 & $0.17 \pm 0.01$ & $0.34 \pm 0.01$ & $0.79 \pm 0.01$ & $50.70 \pm 0.05$ & $51.03 \pm 0.04$ & $50.85 \pm 0.02$ & $2.08 \pm 0.01$ & $2.55 \pm 0.01$ & $0.44 \pm 0.02$ & 1.60 \\
\hline
\end{tabular}




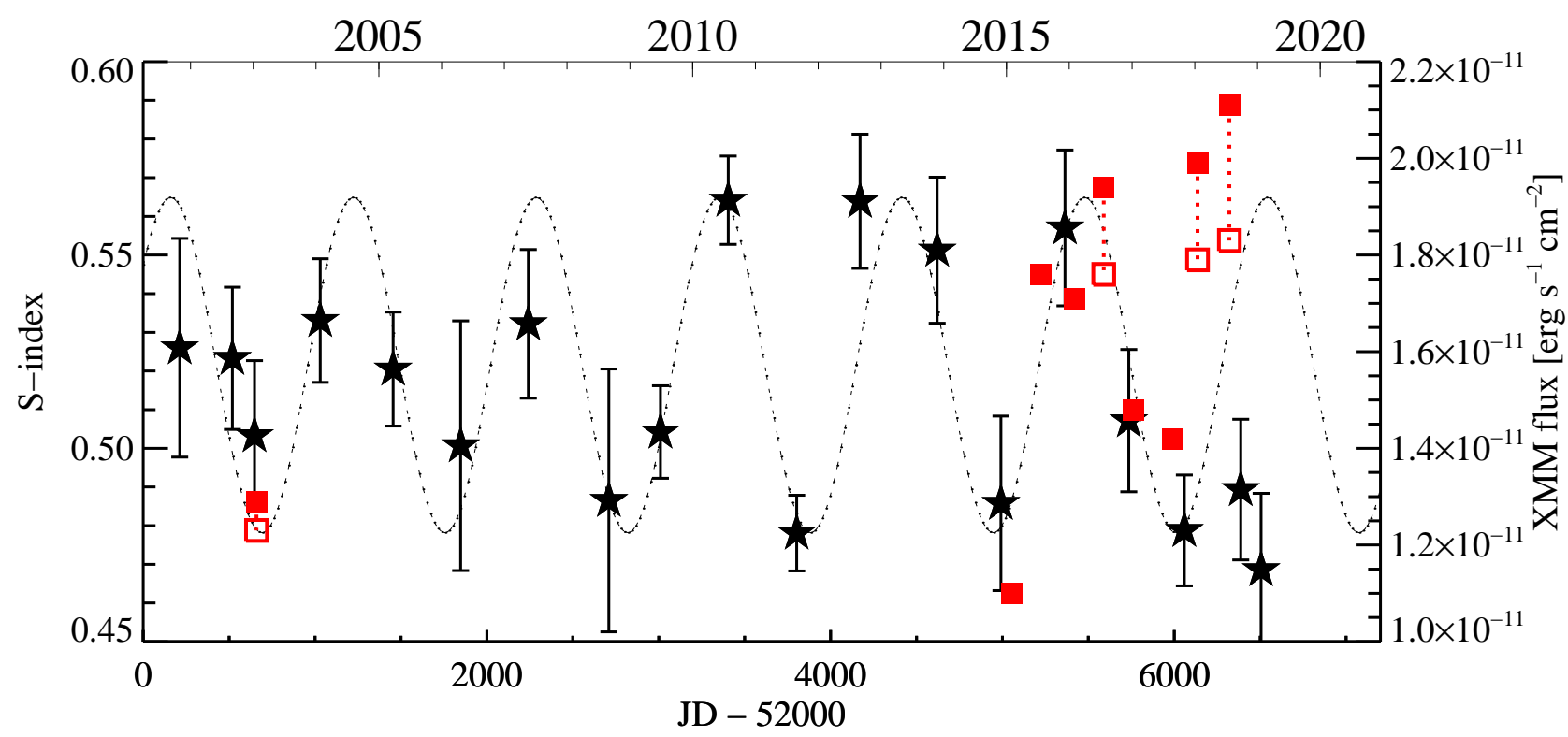

Fig. 3: Long-term lightcurve of $\epsilon$ Eri starting from 2002. The binned Ca II S-index data are plotted with star symbols. The X-ray fluxes are overplotted as red squares. The filled squares refer to the X-ray fluxes calculated over the complete lightcurve of each observation. For those observations with a flare-like short term variability, the X-ray fluxes are also calculated after the removal of the flare and shown as open squares.

mation since its signal-to-noise ratio is lower than that of the $\mathrm{EPIC} / \mathrm{pn}$.

The X-ray data were analyzed with the software SAS (Science Analysis System - version 17.0.0). The standard SAS tools, as described in the SAS Users Guide (de la Calle 2019), were applied to filter event lists of each observation and produce the images.

\subsubsection{EPIC spectra}

We extracted the spectra from the filtered EPIC/pn event lists. We first chose two circular regions to extract the source and the background counts. The source region was centered on the detected source, and the background region on a source-free section of the CCD. We then extracted the spectra from these regions and we scaled them to the chosen area. We generated the redistribution matrix and the ancillary files for each source spectrum, and grouped these together with the spectrum file choosing 40 as minimum number of counts per bin.

Before analyzing the spectra, we corrected them for the so called Out-of-Time events, i.e. EPIC camera photon events registered during the readout of the CCD. In the Full Frame mode and in the Large Frame mode the Out-of-time events are $6.3 \%$ and $0.16 \%$ of the total registered photons, while in the Small Window mode they are $1.1 \%^{4}$. Thus, using SAS we extracted first the spectra of the Out-of-Time events for each observation and then we subtracted them from the respective source spectrum.

All spectra were analyzed with the software xspec (version 12.10; Arnaud 1996). We first considered the merged spectrum, including all observations: we fitted it assuming a 3-T APEC model where the temperatures, the emission measures and the global abundance are allowed to vary. We did not include an absorption component in our model because, due to the small distance of $\epsilon$ Eri, photoeletric absorption is negligible. We obtained

\footnotetext{
4 The percentage of Out-of-Time events for the different observational EPIC modes is reported in the XMM-Newton Users Handbook.
}

as best-fitting parameter of the abundance $0.29 Z_{\odot}$. Similarly, when we fit each single spectrum with the same model, the abundances span a range between $0.2 Z_{\odot}$ and $0.4 Z_{\odot}$. While these values are typical for very young stars (Maggio et al. 2007), from the high-resolution spectrum of $\epsilon$ Eri slightly larger abundances $\left([\mathrm{Fe}] /[\mathrm{H}]=0.5 \pm 0.2\right.$ ) were found (Sanz-Forcada et al. 2004) ${ }^{5}$. A detailed abundance study is beyond the scope of this work. Thus, to minimize the number of free parameters and simplify the subsequent analysis described in Sect. 3, we decided to set the metal abundances on $0.3 Z_{\odot}$ and to keep them frozen during the fitting procedure.

The best-fit spectral parameters of each observation are summarized in Table 2. The associated errors were found with the xspec command error, that determines the confidence interval for the model parameters within a confidence region that we chose equal to $90 \%$. We calculated the fluxes in the soft energy band $0.2-2 \mathrm{keV}$, the luminosities $L_{X}$ and the emission-measure weighted average temperatures as

$T_{\mathrm{av}}=\frac{\sum_{i=1}^{3} k T_{i} \cdot E M_{i}}{\sum_{i=1}^{3} E M_{i}}$.

Fig. 3 shows the resulting long-term X-ray lightcurve together with the $\mathrm{Ca}$ II S-index and the sinusoidal fit resulting from the Lomb-Scargle analysis of the S-index. Here, we binned the Ca II measurements of Fig. 1 for clarity ${ }^{6}$.

Clearly, the X-ray fluxes in Fig. 3 follow the sinusoidal fit to the Ca II variability until late 2017 , providing evidence of an Xray activity cycle. However, the two observations of 2018 show an enhanced X-ray flux. In the next section we show that these observations are affected by short-term variability.

\footnotetext{
5 We note that all these values refer to the coronal abundances which are measured with respect to the solar photospheric abundance.

${ }^{6}$ We divided the $S_{\mathrm{MWO}}$-index set in sub-sets, each of them with a length corresponding to an observing season; the vertical bars are the standard deviation of each sub-set.
} 


\subsubsection{EPIC lightcurves}

EPIC/pn lightcurves were generated in the soft energy band $0.2-$ $2 \mathrm{keV}$ for a time bin size of $300 \mathrm{sec}$.

We systematically analyzed the lightcurves of each individual observation for variability, using the software $R$ and its package changepoint (Killick \& Eckley 2014). A changepoint is denoted as the time at which a significant change of the count rate is present. This tool allows us to identify multiple changes in mean and variance of the count rates within each observation. In Appendix A the lightcurves of all observations and their segmentation are shown. According to this analysis, four out of nine observations show short-term variability. These observations are the ones from January 2003, July 2016, January 2018 and August 2018. The lightcurves of January 2003 and July 2016 show a sudden increase in the count rate resembling the shape of a stellar flare event: in particular the one of 2003 shows most likely the decay phase of a flare. We thus decided for these observations to extract the spectra in the segment of the lightcurve with the lowest count rate and repeat the spectral fitting. The lower Xray fluxes resulting from this analysis are also plotted in Fig. 3 as open red squares.

\section{The corona of $\epsilon$ Eri in the context of solar EMD}

Here we aim at describing the XMM-Newton/EPIC spectrum of $\epsilon$ Eri and its evolution throughout its X-ray cycle in terms of solar emission measure distributions (EMDs).

In the context of the study of the "Sun as an X-ray star" (Orlando et al. 2000; Peres et al. 2000; Reale et al. 2001; Orlando et al. 2001), Orlando et al. (2000) generated a grid of the temperatures $T$ and the emission measures $E M$ of individual pixels from Yohkoh/SXT images of the Sun taken in two different filters during the 1990's. Combining the results over the full surface of the Sun, a "whole-Sun" $E M(T)$ distribution (EMD) was constructed. This analysis was extended to studies of both spatially and temporally distinct observations of the Sun in subsequent articles of the paper series, that here are briefly summarized.

While the analysis of Orlando et al. (2000) was restricted to a single observing date (namely January 6th, 1992), Peres et al. (2000) have extended the study to three different dates, spanning the full range of the solar activity cycle. That work showed that the solar EMD changes strongly throughout the cycle. Reale et al. (2001) derived the EMDs of flaring events (FLs): they chose eight flares, from weak to intense ones (i.e. from class $\mathrm{C}$ to class $\mathrm{X}$ ), as representative of the flaring Sun, and they analyzed separately their rise, peak and decay phase. Orlando et al. (2001) defined three types of solar coronal structures based on the intensity measured in the Yohkoh/SXT images. The full range of measured intensities was assigned to quiet regions (the background corona, BKCs), active regions (ARs) and cores of active regions (COs) in order of increasing intensity. This classification was verified to closely correspond to the distinct spatial structures seen in the images. The EMDs were constructed for each of these type of structures separately during the whole solar cycle. It was, thus, noticed that during the minimum of the cycle the dominant contribution to the EMD comes from the BKCs, while during the maximum from the ARs. Finally, Orlando et al. (2004) examined the temporal evolution of the EMDs of only one visible $\mathrm{AR}$ and one $\mathrm{CO}$ on the solar surface in time-steps of one day and spanning Yohkoh/SXT observations across nearly two months.
The various solar EMDs obtained from these studies were converted into synthetic X-ray spectra using the MEKAL code (Mewe et al. 1986; Kaastra 1992; Mewe et al. 1995), as described by Peres et al. (2000). These spectra can be folded through the instrumental response of non-solar instruments, such as ROSAT, ASCA, XMM-Newton. The final products of the "Sun as an X-ray star" studies were simulated X-ray spectra of the Sun that are analogous to stellar observations and that can be treated with the usual methods of X-ray analysis of the chosen non-solar instrument.

Favata et al. (2008) and Orlando et al. (2017) had applied this study to the XMM-Newton spectra of the star HD 81809 , with the aim of using the Sun as a template to link the stellar coronae physics to the standard solar model. They combined the solar EMDs extracted for ARs, COs and FLs and scaled them to the size of HD 81809. By varying the coverage fractions of each solar structure on the surface of the star, they had then built a grid of EMDs to artificially reproduce a solar-like corona with the physical characteristics of HD 81809. Subsequently, they extracted X-ray spectra from the grid to be compared with the observed EPIC spectra of HD 81809 to interpret the evolution of the X-ray cycle in terms of coronal structures. This can only be achieved by a spectral analysis since, in contrast to the Sun, the magnetic structures on the artificial solar-like corona are not spatially and temporally resolved and diagnosed. Here, we apply the same method to $\epsilon$ Eri.

\subsection{Standard solar coronal structures}

Among the coronal structures described and analyzed in the study of the "Sun as an X-ray star", we used ARs, COs and FLs. The emission measure of $\epsilon$ Eri results to be higher than the solar one. Thus, we ignored the BKCs because, among all types of structures, they have the lowest intensity and a high percentage of coverage would be required to produce a significant contribution to the total EMD. This would considerably reduce the available surface for the other magnetic structures, that instead, given their higher intensity, are better suited to reproduce the $L_{X}$ of $\epsilon$ Eri. Moreover, the solar BKCs have low temperatures that are only marginally covered with XMM-Newton. Drake et al. (2000) showed that the EMD of $\epsilon$ Eri at temperatures representative of solar BKC $\left(T \sim 10^{6} \mathrm{~K}\right)$ obtained from Extreme Ultraviolet Explorer (EUVE) observations is significantly greater than that of the full Sun. In Sect. 4 we will show that the EMD of $\epsilon$ Eri derived with $X M M$-Newton at these temperatures well matches the one obtained from the EUVE.

For the $\operatorname{EM}(T)$ distribution of ARs and COs, we took into account the time-averaged distribution of these structures presented by Orlando et al. (2004), i.e. the average of the evolution of only one solar active region and one core observed by Yohkoh in 1996, from its emergence to its decay. For the flare $E M(T)$ we considered the time-averaged distribution of the eight flares discussed in Reale et al. (2001). The assumptions to derive the flaring contributions are the same given in Orlando et al. (2017), which take into account the differential flare energy distribution $N(E) \propto E^{\alpha} d E$ of the Sun with index $\alpha=1.53$ (see Orlando et al. 2017 for details).

We produced the EMDs for unit surface area for the metal abundance used in the fits of the EPIC/pn spectra of $\epsilon$ Eri, i.e. $0.3 Z_{\odot}$. However, the EMDs of each solar structure had been extracted considering solar metal abundances. We thus scaled the EMDs by a factor of $1 / 0.3$ that compensates for the reduced radiative losses associated with lower abundances. Then we obtained the EMDs of each magnetic structure by multiplying the 

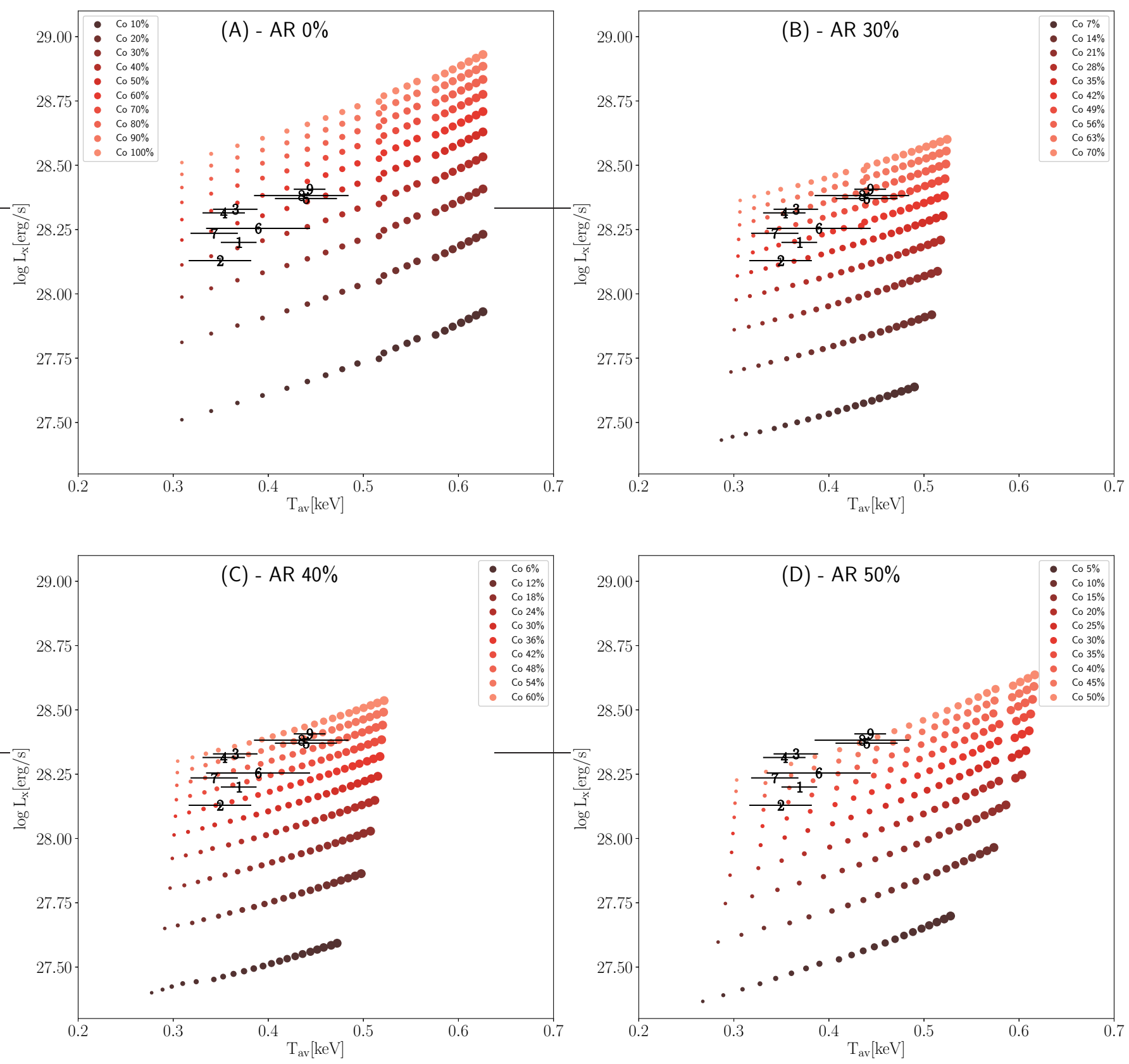

Fig. 4: Four combinations of standard coronal structures explored: (a) AR equal to 0\% and COs varying between 10\% and $100 \%$; (b) AR equal to $30 \%$ and COs varying between $7 \%$ and $70 \%$; (c) AR equal to $40 \%$ and COs varying between $6 \%$ and $60 \%$; (d) AR equal to $50 \%$ and COs varying between $5 \%$ and $50 \%$. For all the combinations the FLs are allowed to vary between $0 \%$ and $2 \%$ of the CO area fraction. Increasing CO coverage is represented by darker color, while increasing FL fraction is represented by increasing symbol size. The overplotted numbers are the observed values of $\epsilon$ Eri and are chronologically ordered.

EMDs per unit surface area by the stellar area of $\epsilon$ Eri that might be covered by these structures.

The task is now to determine the relative contribution of ARs, COs and FLs to the X-ray emission of $\epsilon$ Eri. We constructed thus a grid of $E M(T)$ distributions where each grid point represents a different percent coverage of the coronal structures on the surface of $\epsilon$ Eri. We synthesized the EPIC/pn spectra for each grid point of the composite $E M(T)$ characterized by different fractional contributions of the three magnetic structures and assuming abundances $0.3 Z_{\odot}$. We analyzed each synthetic spectrum in the same way as we had done for the observed spectra of $\epsilon$ Eri, i.e. we fitted them with a 3-T thermal APEC model with metal abundance fixed at $0.3 Z_{\odot}$ (Sect. 2.2). We then calculated for each grid point the X-ray luminosities $\left(L_{X}\right)$ and the average temperatures $\left(T_{\mathrm{av}}\right)$ as in Sect. 2.2.

In Fig. 4a-d we explore how different combinations of ARs, COs and FLs affect the derived $L_{\mathrm{X}}$ and $T_{\mathrm{av}}$. In these panels the results for the synthetic spectra are represented by dots. The $L_{X}$ and the $T_{\mathrm{av}}$ derived for $\epsilon$ Eri are overplotted onto this grid with numerical symbols, following the chronological order of each observation. In Fig. 4a the AR coverage fraction is set to $0 \%$, while the COs are varying between $10 \%$ and $100 \%$ as shown in 


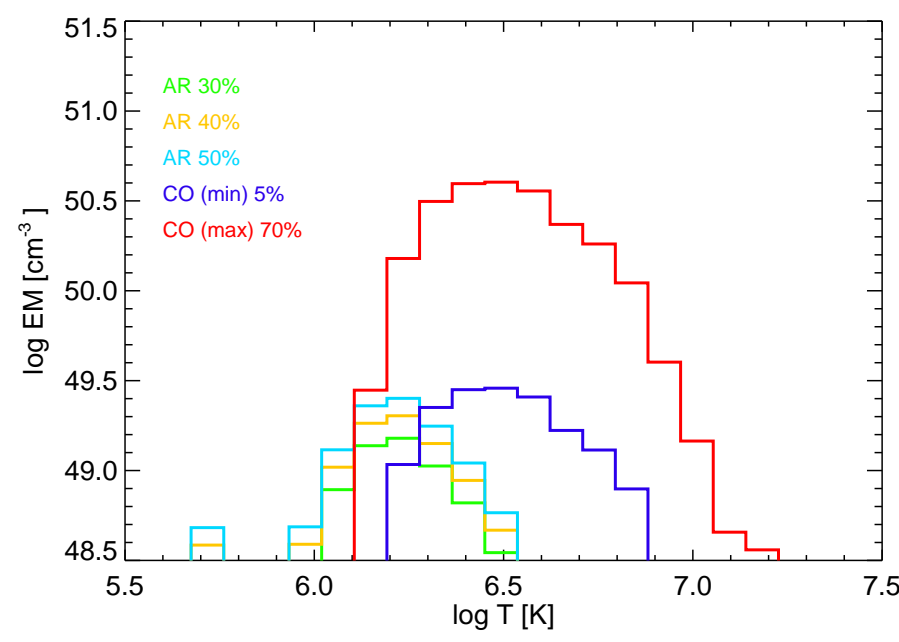

FIG. 5: EMDs of the three combinations of ARs shown in Fig. 4b-d scaled to the surface area of $\epsilon$ Eri. The EMD of ARs are plotted in green, in yellow and in cyan for each investigated coverage fraction. The EMD of COs are also overplotted in blue for the minimum of the considered coverage fraction and in red for the maximum.

the inset. The FLs vary between $0 \%$ and $2 \%$ of the area covered by the COs, with a step size of $0.1 \%$. The symbol size and the color in the plots represent, respectively, the variation of the percentage of FLs and COs. It can be seen that an increase in the coverage fraction of the FLs influences mainly the average temperature, whereas an increase in the coverage fraction of the COs influences the luminosity. We notice that the observed $L_{X}$ and $T_{\text {av }}$ of $\epsilon$ Eri are well reproduced with a surface coverage with COs that does not exceed $60 \%$.

The impact of adding AR coverage greater than $0 \%$ can be seen in panels b-d. Obviously, the sum of the coverage fraction of ARs and COs can not exceed $100 \%$. To set an upper limit for the contribution of ARs and COs, we explored three different combinations of coronal structures around the percentage of the COs of $60 \%$. We chose ARs equal to $30 \%$ and COs varying between $7 \%$ and $70 \%$ (Fig. 4b); ARs equal to $40 \%$ and COs varying between $6 \%$ and $60 \%$ (Fig. $4 \mathrm{c}$ ); ARs equal to $50 \%$ and COs varying between $5 \%$ and $50 \%$ (Fig. 4d). For all the combinations shown in Fig. 4, the flares are allowed to vary between $0 \%$ and $2 \%$ of the percentage of COs. In Fig. 5 the EMDs of the ARs for each combination are shown, together with the minimum and the maximum of COs that we considered in all the combinations.

As can be seen from Fig. 4b-d, all three combinations can potentially reproduce our observational data. We reject the combination of Fig. $4 \mathrm{~d}$ because the $L_{\mathrm{X}}$ of the synthetic spectra cover the data only at the limit. The other combinations reproduce the observations. However, we can set only an upper limit for the coverage fraction of ARs. As matter of fact, the emission measure of the ARs is lower than that of the COs per unit of covered surface, as is evident in Fig. 5 where the EMD of the minimum coverage fraction of COs, i.e. 5\%, has an emission measure comparable to the maximum coverage fraction of ARs, i.e. $50 \%$. Thus, any reasonable coverage fraction of ARs provides only a small contribution to the overall EMD, and the difference between this contribution for different AR percentage is small (see Fig. 5 for $30 \%, 40 \%$ and $50 \%$ of AR) such that we can not set a lower limit on the coverage of ARs. Thus, the grid chosen for further analysis is the combination with a coverage of ARs equal to $40 \%$, a coverage of COs that varies from $6 \%$ to $60 \%$ and
FLs varying from $0 \%$ to $2 \%$ (Fig. 4c), without excluding that the surface can also be covered with a lower fraction of ARs.

For a more detailed investigation of the compatibility between the observed spectra of $\epsilon$ Eri and the synthetic spectra derived from the solar $E M(T)$, we proceeded to a comparison of the individual spectral best fit parameters, i.e. the three temperatures and the three emission measures.

In Fig. 6 we plot the best-fit parameters obtained from the synthetic spectra for the chosen combination of solar structures (case Fig. 4c), together with the ones of the best fit to the observed spectra of $\epsilon$ Eri (see Table 2). Analogous to Fig. 4, the variation of the colors represents the percent coverage of COs, while the symbol sizes the percentage of FLs. As can be seen from Fig. 6, an increase of COs on the surface influences the two lower-temperature components of the spectral model, while an increase of the flaring coverage influences mostly the third component, i.e. the hottest one.

To properly compare the best-fit parameters of the synthetic and the observed spectra, we introduced in the procedure with which we extract the synthetic spectra from the solar-based EMDs a statistical randomization, i.e. Poisson statistics, typical of the satellite's effective area. In this way we simulate the synthetic spectra as if they were actual XMM-Newton observations. Moreover, to refine further the comparison, for each grid point we performed a Monte-Carlo simulation: we generated 1000 randomized spectra for each grid point, so that each combination of magnetic structures is represented by 1000 spectra. We analyzed for each grid point each set of 1000 synthetic spectra in the same way as we had done for the observed spectra of $\epsilon$ Eri, i.e. we fitted them with a 3-T APEC model with metal abundance fixed at $0.3 Z_{\odot}$ (Sect. 2.2). The result of having introduced statistical noise in the simulated spectra is that each combination of magnetic structure is not univocally represented by only one set of values for $k T$ and $E M$, but by 1000 values with a pseudorandom distribution. We demonstrate this in Fig. 7 where, as an example, we show a zoomed plot of the first and the third component of the temperatures. In the plot the black circles are the medians of the 1000 best fit values for each combination of magnetic structures in the range of temperatures displayed in the zoom. For one specific combination (COs $42 \%$ and FLs $0.4 \%$ ) we highlight in red its median and, as an example, we overlay in blue the best-fitting temperatures for all 1000 representations of this AR-CO-FL combination. As error bars on the (red) median we adopt the percentile at $10 \%$ and $90 \%$ of the 1000 values. Evidently, these 1000 values define a significant spread. If this spread is ignored and a one-by-one comparison between observations and synthetic spectra is performed, the result overestimates the truly achievable accuracy in the determination of the best matching model grid point. In other words, the comparison with the synthetic spectra should take into account all the 1000 representations obtained for each grid point.

Next we describe how we performed this match between observed and synthetic model parameters. For clarity, in the following we denote the six spectral parameters $\left(k T_{1,2,3}\right.$ and $\left.\mathrm{EM}_{1,2,3}\right)$ by $P_{i}$, with $i=1, \ldots, 6$. To find the best-fitting combination of magnetic structures, we matched the best fit parameters $P_{i}^{o b s}$ for each observation with each of the corresponding synthetic parameters $P_{i, j, k}^{s y n}$ derived for the 1000 sets (henceforth labeled with $j$ ) of all 201 different combinations of solar regions (grid points; henceforth labeled with $k$ ), where $j=1, \ldots, 1000$ and $k=1, \ldots, 201$. As selection criterion we evaluated the following six equations:

$P_{i}^{o b s}-\Delta P_{i}^{o b s} \cdot \sigma \leq P_{i, j, k}^{s y n} \leq P_{i}^{o b s}+\Delta P_{i}^{o b s} \cdot \sigma$ 

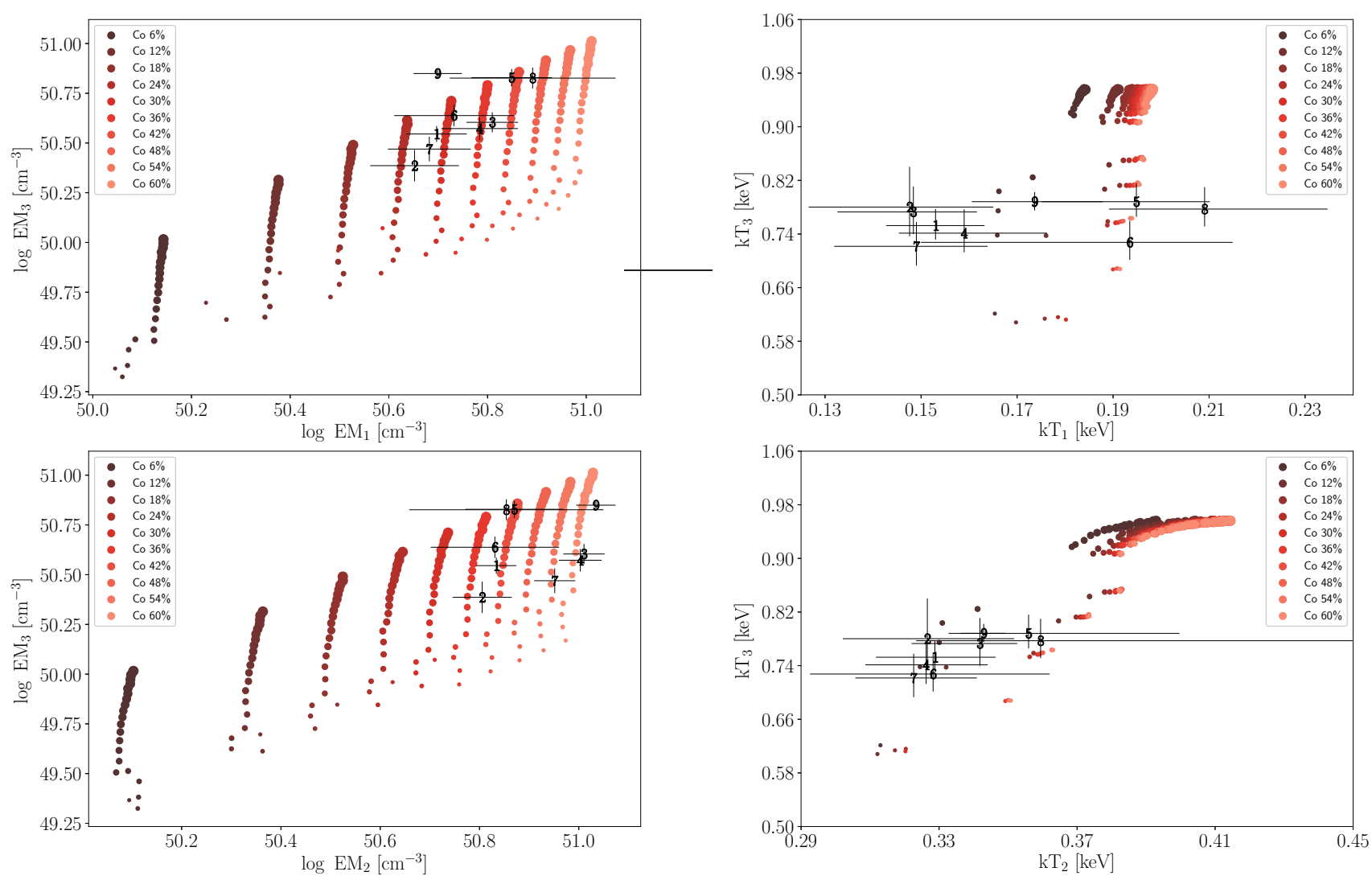

Fig. 6: Parameters of the best-fittting 3T-model for the individual observations of $\epsilon$ Eri (numbers) with overplotted the best-fit parameters obtained from the synthetic spectra for the grid from Fig. 4c. On the left, the emission measures. On the right, the temperatures. Colors and symbol sizes are the same as in Fig. 4.

with a unique parameter $\sigma$ for all six spectral parameters. $\sigma$ defines thus the global confidence range of the match between observed values $P_{i}^{\text {obs }}$ and synthetic values $P_{i, j, k}^{s y n}$ with $i=1, \ldots, 6$. We then picked for each of the 1000 sets $(j)$ of combinations of solar regions as best-matching model the one among all grid points $(k)$ that provides the smallest value for $\sigma$. The result of this procedure are 1000 best-fitting representations (for each observation).

The spread of the 1000 representations in terms of spectral parameters (exemplified in Fig. 7) thus translates into a range of selected best-fitting combinations of AR, CO, and FL coverage. We define as final best-matching combination of ARs, COs and FLs the median of the 1000 values retrieved with our selection procedure and we associate an uncertainty on this result as the $10 \%$ and $90 \%$ quantile of the 1000 values (red bars in the example of Fig. 7).

The EMDs corresponding to the models selected with this procedure as the best match to the observations are shown in Fig. 8. The total contribution of all coronal structures is the gray distribution, whereas the blue is the contribution from ARs, the green from COs and the red from FLs. The median area coverage fraction for each of the three types of structures, with the associated errors, is given in the legend of the panels. In addition, the best-fit parameters of the 3-T model fitted to the observed spectra of $\epsilon$ Eri with the associated errors from Table 2 are plotted (black circles). Finally, in red we show the medians of the best-fit parameters of the 1000 best matching spectra, and the percentiles at $10 \%$ and $90 \%$ of these representations, denoting the minimum and the maximum of the error bars respectively.

In Fig. 9 we present a summary of the correspondence between the observed and modeled 3-T best-fitting parameters: we plot the ratio between the best-fit parameters of $\epsilon$ Eri and the medians of best-fit parameters of the corresponding selected $E M(T)$. The ratios of the temperatures result systematically $<1$, i.e. the temperatures of the selected synthetic spectra are higher than the ones of the observed spectra. While the first and the second component of the emission measures give also a ratio $<1$, the $E M_{3}$ of the selected model is drastically lower than the observed values. Therefore, among the six parameters, the third component of emission measure shows the most drastic discrepancy (see also the discrepancy between the black circles and the red triangles in Fig. 8).

To summarize, the grid of solar coronal structures with ARs fixed on $40 \%$, COs between $6 \%$ and $60 \%$ and FLs between $0 \%$ and $2 \%$ is able to reproduce the X-ray luminosity and the average coronal temperature of all observed EPIC/pn spectra of $\epsilon$ Eri 


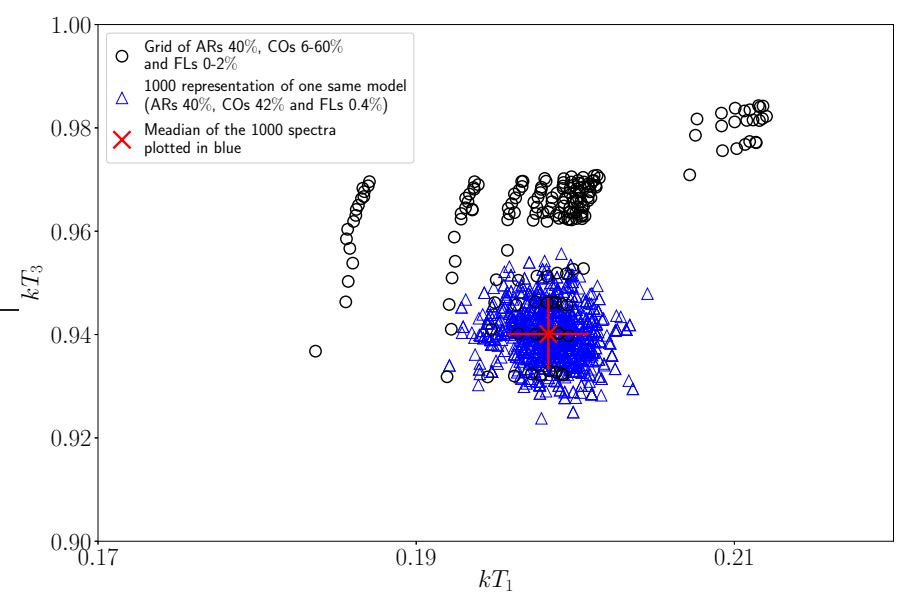

Fig. 7: Example showing the effect of the Poisson statistics on the retrieved spectral parameters $\left(k T_{1}\right.$ vs. $\left.k T_{3}\right)$ of the synthetic spectra of the grid with ARs $40 \%$, COs 6-60\% and FLs $0-2 \%$. Black circles - medians of the 1000 representations of each grid point; Blue triangles - 1000 best fit parameters representing one specific combination of magnetic structures (ARs $40 \%$, COs $42 \%$, FLs $0.4 \%$ ); Red cross - median of the 1000 representations (plotted in blue) of the model with COs $42 \%$ and FLs $0.4 \%$ that we chose to highlight as an example.

(Fig. 4c), but the $\operatorname{EM}(T)$ structure does not match very well its spectral shape (Fig. 6, Fig. 8, Fig. 9).

\subsection{Modified solar coronal structures}

Here we investigate if modifications to the solar coronal EMD can reproduce the observed X-ray spectra of $\epsilon$ Eri better.

As shown in Sect. 3.1, the most severe discrepancy between synthetic and observed spectra is given by the third component of the $E M$ : the synthetic parameters $E M_{3}$ are systematically lower than the observed ones (Fig. 9). The standard EMD previously tested comprises values of $E M_{3}$ sufficiently high to be compatible with the observed values (see Fig. 6). However, high values of $E M_{3}$ in the grid correspond to high values of $k T_{3}$, that do not agree with the observed $k T_{3}$, and consequentially are rejected by our selection procedure. Thus, in order to find models that match both the temperatures and the emission measures of the hottest spectral component, we decided to modify the flare $E M(T)$ because FLs are the structures that influence most strongly the hottest component.

We examined various versions of flare $\operatorname{EM}(T)$ as discussed in the following. These $E M(T)$ and the original ones described in Sect. 3.1 are compared in Fig. 10.

\subsubsection{Contribution of flares at soft and hard energies}

All the flares presented in Reale et al. (2001) were observed by Yohkoh with the two hardest SXT filters, that are sensitive to plasma around and above $10^{7} \mathrm{~K}$. The data published by Reale et al. (2001) comprises two flares of class M that were also observed with softer filters. Soft filter data were published for only one of these two flares, and showed that a contribution from plasma at lower temperatures is important. The soft emission from flares modifies the flare $E M(T)$ by adding an extended low-temperature tail. We thus replaced the flare $E M(T)$ from Sect. 3.1 with the average between these two M class flares, including the soft emission. The new flare EMD is the blue distribution in Fig. 10.
We then built a new grid of EMDs with the same ARs and COs of the previous analysis, i.e. $40 \%$ of ARs and COs varying between $6 \%$ and $60 \%$, and we again set the percentage of FLs between $0 \%$ and $2 \%$ of the percentage of COs. We extracted and fitted the corresponding spectra as in Sect. 3.1. Fig. B.1 shows the EMDs of the corresponding synthetic spectra selected for each observation, analogous to Fig. 8 and with the same meaning of the symbols. Some of the observations now require a smaller coverage fraction of COs because of the additional soft emission of the flare $\operatorname{EM}(T)$. In Fig. 11 we plot the ratio between the bestfit parameters of $\epsilon$ Eri and the medians of the best-fit parameters of the corresponding selected $E M(T)$ (blue circles). The discrepancies between observed and synthetic parameters are somewhat smaller than in the previous case but they show the same pattern (compare blue symbols in Fig. 11 with Fig. 9). In particular for the third component of the emission measure the differences are still large.

\subsubsection{Flares in the decay phase at soft and hard bands}

We built another flare EM(T) by averaging the two flares of class M observed with the soft filters and the hard filters, but only during their decay phase (green distribution in Fig. 10). We adopted the same coverage fraction of ARs and COs of the previous analysis, but we set the percentage of FLs to vary between $0 \%$ and $10 \%$ within the percentage of COs. A higher coverage fraction of FLs than the one we adopted previously is required to reproduce the observed $T_{\mathrm{av}}$, since this flare EMD has a lower emission measure and a lower temperature compared to the previous EMDs, as can be noticed from Fig. 10.

We then proceeded on the extraction and fitting of the synthetic spectra and on the selection procedure adopted in Sect. 3.1. The EMDs of the corresponding spectra selected for each observation are shown in Fig. B.2. These EMDs are composed of significantly lower $\mathrm{CO}$ coverage fraction than the previous ones and the FL fraction is up to $\sim 5 \%$. The observed and simulated spectral parameters are now in much better agreement. This is also seen in Fig. 11, where the ratios between the best-fit parameters of $\epsilon$ Eri and the medians of the 1000 best-fit parameters of the corresponding selected $E M(T)$ are plotted as green circles. A major improvement is obtained for the hot component, where the ratios for both $k T_{3}$ and $E M_{3}$ are now much closer to 1. The parameters representing the other two spectral components also show somewhat better agreement than before. This suggests that flare plasma with lower temperature, such as solar flares during the decay phase, better describes the phenomena in the corona of $\epsilon$ Eri.

\subsubsection{Flare in the decay phase at hard band}

As final test, we built a new grid of EMDs, using a flare EM(T) of the two class $M$ flares observed only with the hard filters and limited to their decay phase (red distribution in Fig. 10). The EMDs of the corresponding spectra selected for each observation are shown in Fig. B.3. Compared to the flares in decay that included soft emission (Sect. 3.2.2), the FL coverage is smaller and more COs are present. The red circles in Fig. 11 are the ratios between the best-fit parameters of $\epsilon$ Eri and the medians of the 1000 bestfit parameters of the corresponding selected $E M(T)$. The results are very similar to the previous analysis in Sect. 3.2.2. The red symbols in Fig. 11 are comparable to the green ones, i.e. we can not distinguish which of these two flare distributions better matches the EMD of $\epsilon$ Eri. 

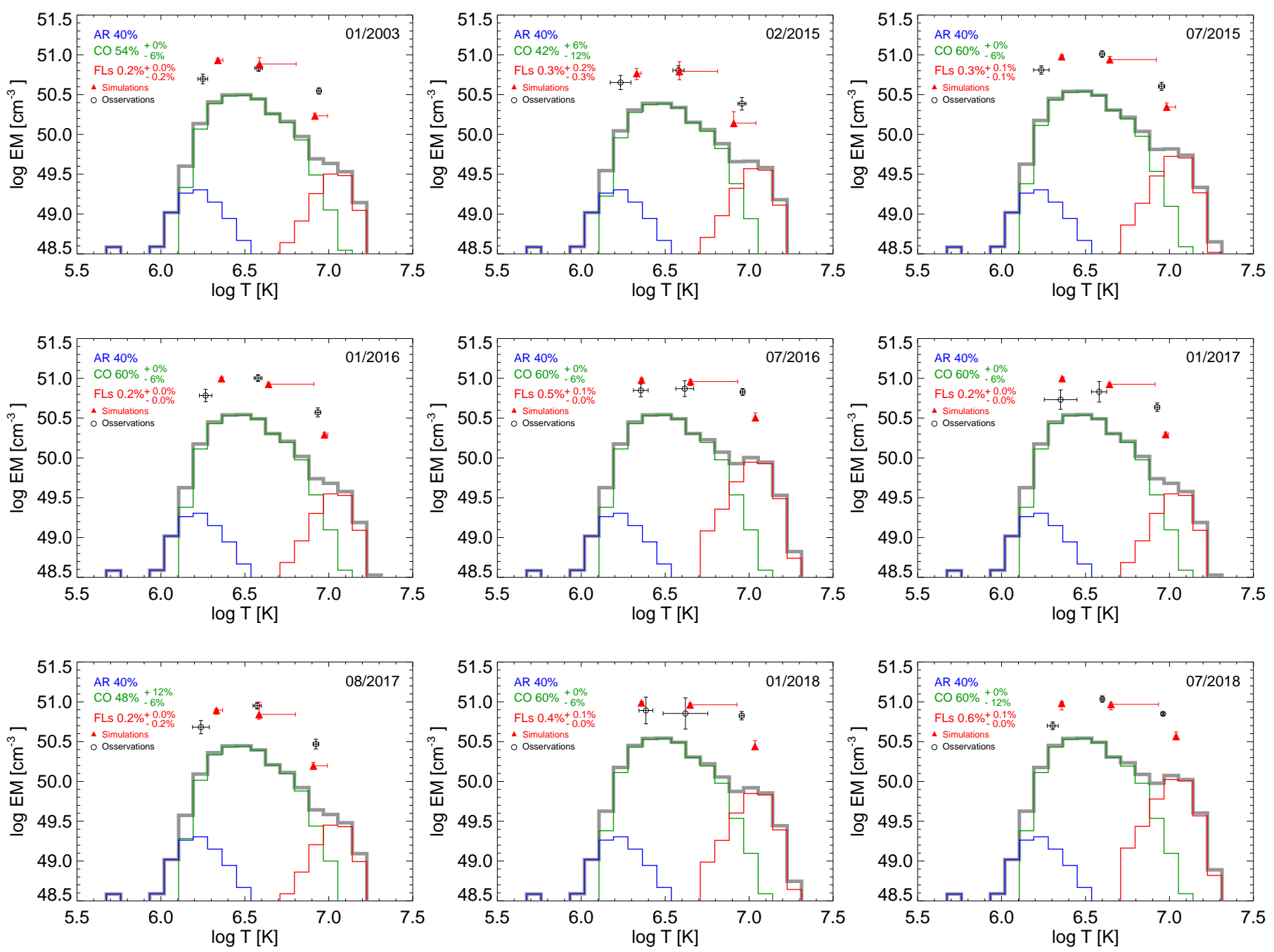

Fig. 8: Solar coronal EMD (composed of ARs, COs and FLs) that best matches the spectral parameters of $\epsilon$ Eri. blue line - AR contribution; green line - CO contribution; red line - FL contribution; grey line - total EMD of the chosen model. Each observation is represented by a different fraction of surface coverage with AR, CO and FL selected as described in Sect. 3.1. Red triangles - medians of the 1000 best-fit parameters of the selected synthetic spectra; black dots - best-fit parameters of the observations.

\subsection{Results}

To summarize, among the four different EMDs for solar-like flares, the last two tests involving flares in the decay phase best represent the observed EMD of $\epsilon$ Eri. In Sect. 4 and Sect. 5 we provide an interpretation of this finding.

\section{Discussion}

We have detected for the first time the X-ray cycle of the young solar-like star $\epsilon$ Eri. Our X-ray monitoring, started in 2015, is longer than the full duration of the known chromospheric Ca II $\mathrm{H} \& \mathrm{~K}$ S-index cycle. To validate the newly identified X-ray cycle we have re-analyzed the Ca II H\&K S-index variation based on data from 2002 to 2018 . The analysis revealed a periodic signal of $2.92 \pm 0.02 \mathrm{yr}$, in agreement with past results found on a data set including also historical data (Metcalfe et al. 2013). While until 2017 the X-ray variability follows the Ca II variability, starting in early 2018 these two activity measures seem to disagree. The chromospheric cycle does not reach the ex- pected maximum, whereas the last X-ray observations show an enhanced X-ray flux. Ongoing continued monitoring of both activity indicators will reveal whether a qualitative change of the cycle is taking place.

To put $\epsilon$ Eri in context to other stars with activity cycles, in particular cycles detected in the X-ray band, we show in Fig. 12 the relationship between the cycle period $P_{\text {cyc }}$ and the rotational period $P_{\text {rot }}$, where the updated historical Ca II cycles (Böhm-Vitense 2007; Brandenburg et al. 2017) are plotted together with the stars with known X-ray activity cycles detected by XMM-Newton. The relation between the stellar cycle period, $P_{\text {cyc }}$, and the rotation period, $P_{\text {rot }}$, provides information on the efficiency of the stellar dynamo. In the $P_{\text {cyc }}-P_{\text {rot }}$ diagram, a linear relation is found and two main branches are distinguished: the so-called active (A) branch, where the dynamo may operate on the surface, and the inactive (I) branch, where the dynamo may operate in deeper convective zones (Brandenburg et al. 1998; Saar \& Brandenburg 1999; Böhm-Vitense 2007; See et al. 2016; Brandenburg et al. 2017; Olspert et al. 2018). $\epsilon$ Eri, together 

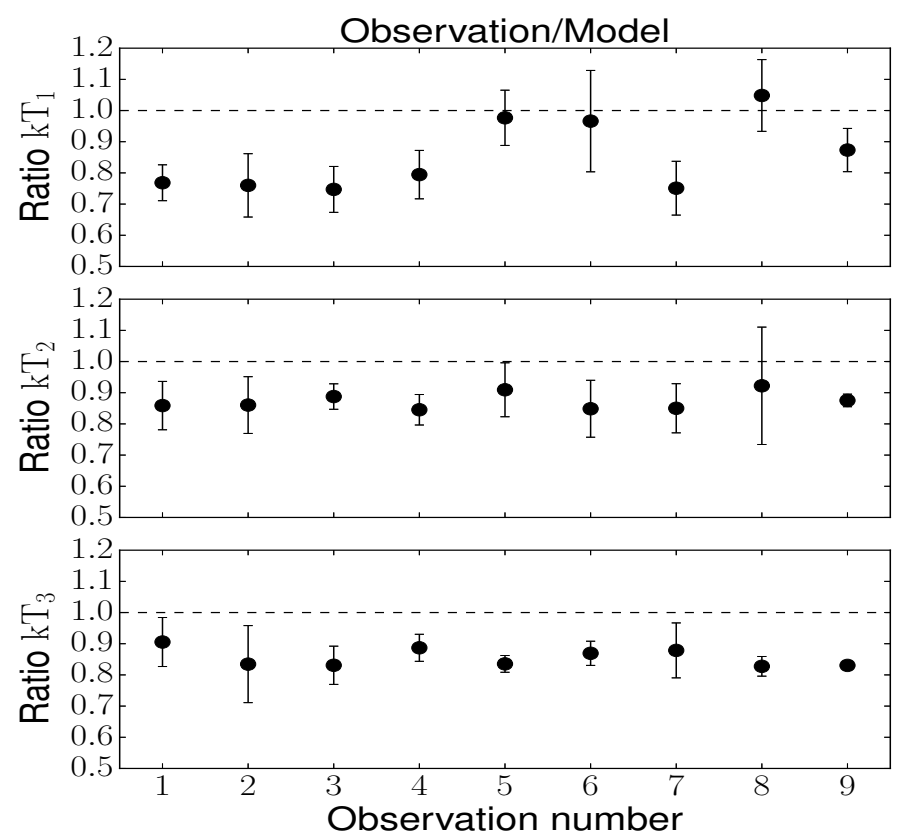
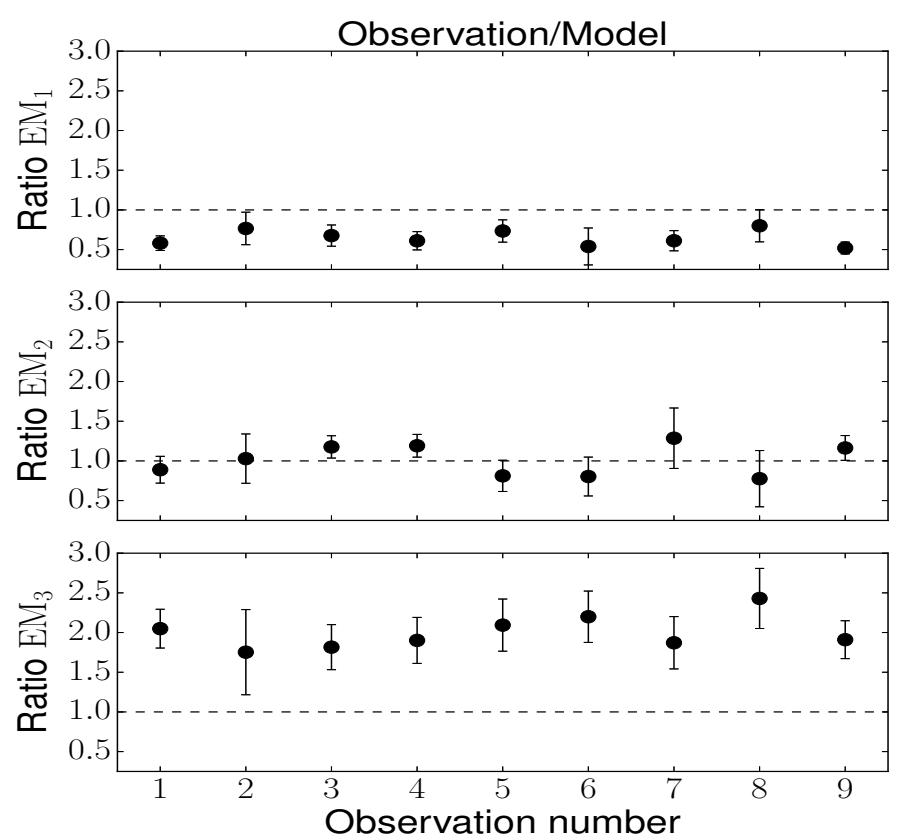

Fig. 9: Ratio of the best-fit parameters of $\epsilon$ Eri over the same parameters selected from the grid with ARs $40 \%$, COs $6-60 \%$ and FLs $0-2 \%$ of COs. The flare EMD is the time averaged distribution of the flares presented by Reale et al. (2001) discussed in Sect. 3.1. The error bars are the errors on the ratios found from error propagation taking into account both the uncertainties of observed best-fitting parameters and of the $10 \%$ and $90 \%$ percentiles of the 1000 selected representations.

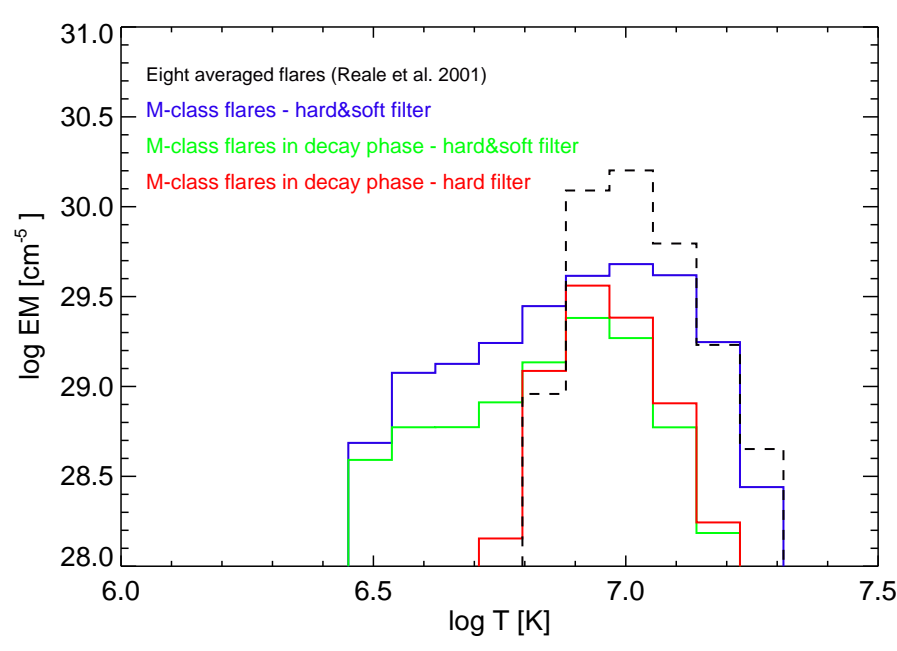

Fig. 10: Flare $\operatorname{EM}(T)$ distributions used to modify the solar coronal EMD, normalized to unit surface. Black distribution - timeaveraged flares presented in Reale et al. (2001) generated according to Orlando et al. (2017); blue distribution - contribution of flares at soft and hard energies; green distributions - contribution of flares in the decay phase; red distribution - contribution of flares at the lowest temperatures.

with $\iota$ Hor, have the shortest X-ray cycles detected so far and are fast rotators, with a rotational period of 11.1 days (Baliunas et al. 1995) and 8.2 days (Sanz-Forcada et al. 2019) respectively. They are thus placed at the bottom of the inactive branch in the $P_{\text {cyc }}-P_{\text {rot }}$ diagram.

In Fig. 13 we show the relation between the X-ray luminosity and the stellar age of the stars with confirmed X-ray cycles. The vertical bars in the plot are the amplitude of the cycles, corresponding to the range between the observed maximum and minimum of $L_{\mathrm{X}}$. From our analysis we found that $\epsilon$ Eri has an aver- age X-ray luminosity $L_{\mathrm{X}}$ of $2.0 \times 10^{28} \mathrm{erg} / \mathrm{s}$, with an amplitude of $1.3 \times 10^{28} \mathrm{erg} / \mathrm{s}$. The values of the other X-ray luminosities and their amplitudes are taken from Robrade et al. (2012) for the $\alpha$ Cen and 61 Cyg systems, from Orlando et al. (2017) for HD 81809 and from Sanz-Forcada et al. (2013) for $\iota$ Hor. Consistent with the decrease of X-ray luminosity with stellar age, the two youngest stars, $\epsilon$ Eri and $\iota$ Hor, have the highest X-ray luminosity of all stars with known X-ray cycles. We notice also that the amplitudes of their cycles have the lowest values.

In this work, we provide a physical explanation for these findings thanks to a new tecnique to interpret the stellar X-ray spectra. This method allows us to examine the evolution of the corona of $\epsilon$ Eri during its X-ray cycle in terms of the EMDs of solar magnetic structures, i.e. active regions, cores of active regions and flares (ARs, COs and FLs). The same method had been applied before only to one star, HD 81809, by Favata et al. (2008) and Orlando et al. (2017).

Compared to HD 81809, the X-ray spectra of $\epsilon$ Eri are of much higher quality and, therefore, they provide more information on the coronal temperature structure. This requires a more sophisticated analysis of the effects of the statistical noise on the accuracy with which the spectral parameters can be constrained. Thus, unlike to the literature studies of HD 81809, we performed a Monte-Carlo simulation for each combination of magnetic structures. As we showed for an example in Fig. 7, the spectral parameters retrieved from fitting the synthetic spectra including random statistical noise show fluctuations that are larger than the typical separation of individual grid points representing specific combinations of ARs, COs and FLs. This leads to a degeneracy in the mapping of spectral parameters $(k T$ and $E M)$ to the magnetic region coverage fraction. Therefore, a oneto-one comparison between the observed X-ray spectra of $\epsilon$ Eri and the synthetic spectra, such as done in previous works on HD 81809 , overestimates the achievable accuracy. We have instead here determined the range of uncertainty associated with each best-fitting model (represented by a specific percentage of 

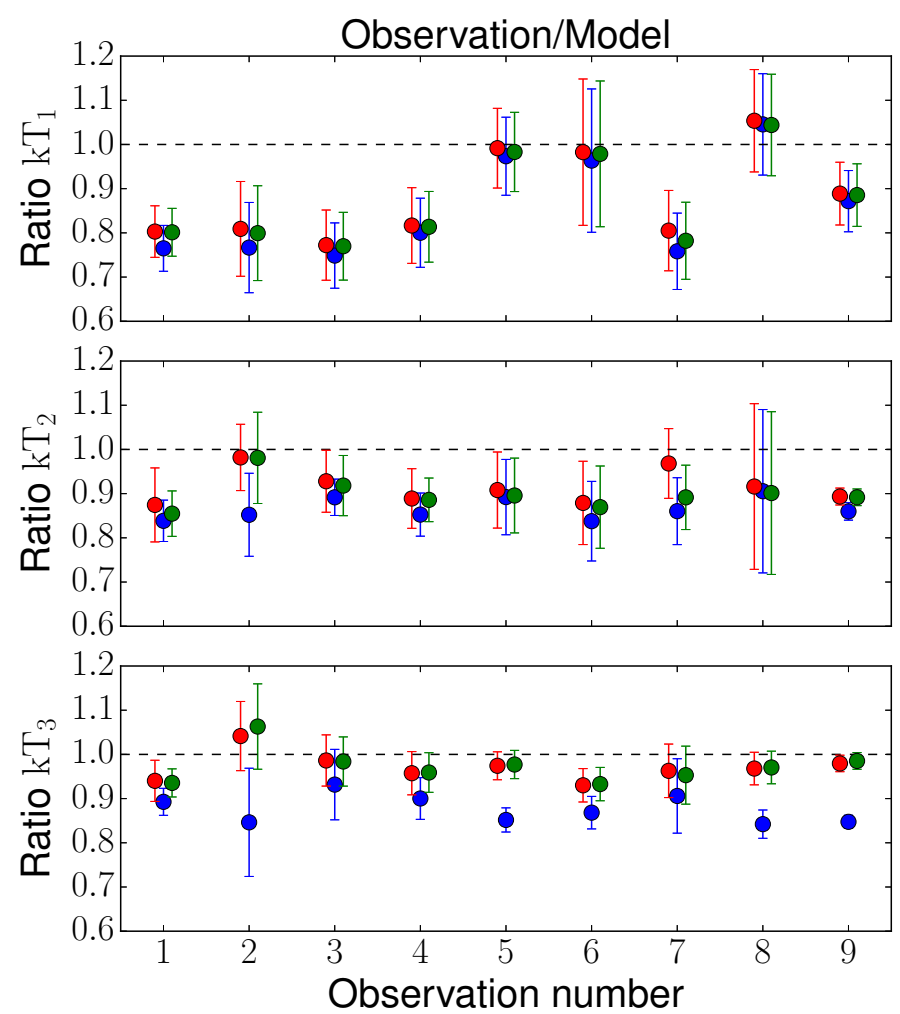
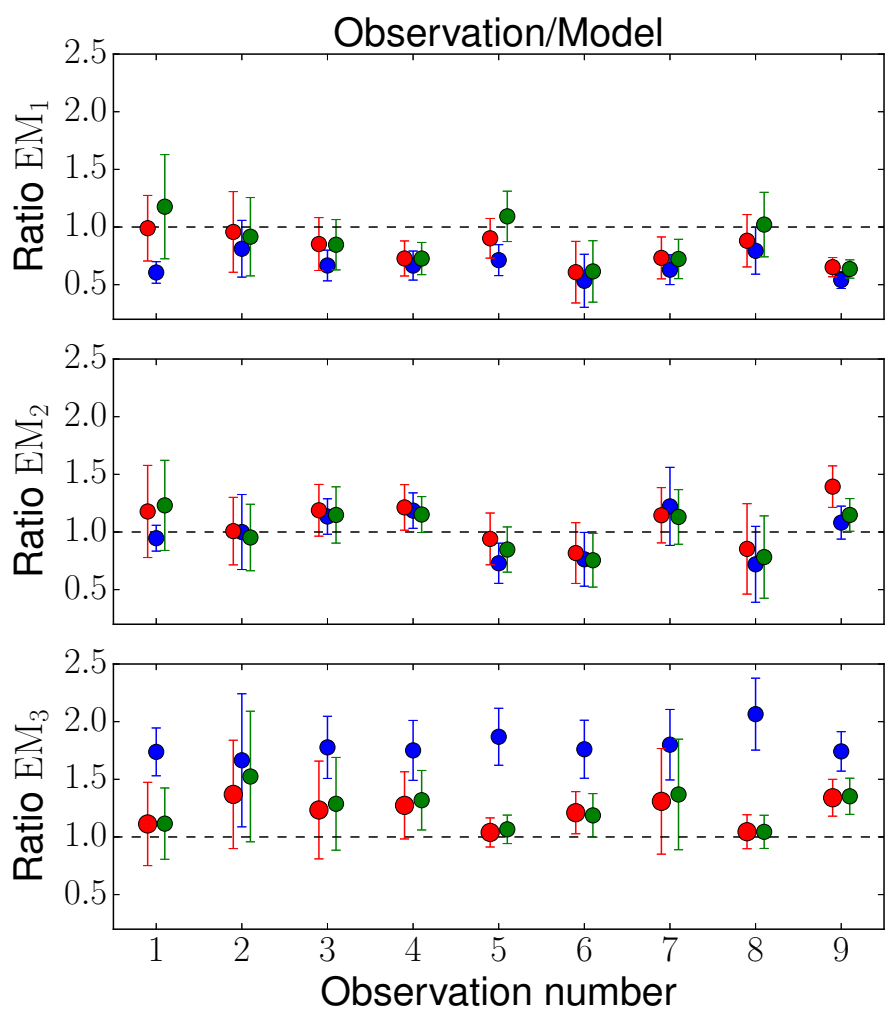

Fig. 11: Ratio of the best-fit parameters of $\epsilon$ Eri over the same parameters selected from the grid with ARs $40 \%$, COs $6-60 \%$. The color code follows Fig. 10. Blue circles - FLs $0-2 \%$ of COs for class M flares at the soft and hard energies; green circles - FLs $0-10 \%$ of COs for class $\mathrm{M}$ flares at the soft and hard energies in the decay phase; red circles - FLs $0-10 \%$ of COs for class M flares at the hard energies in the decay phase. The error bars are the errors on the ratios found from error propagation taking into account both the uncertainties of observed best-fitting parameters and of the $10 \%$ and $90 \%$ percentiles of the 1000 selected representations.

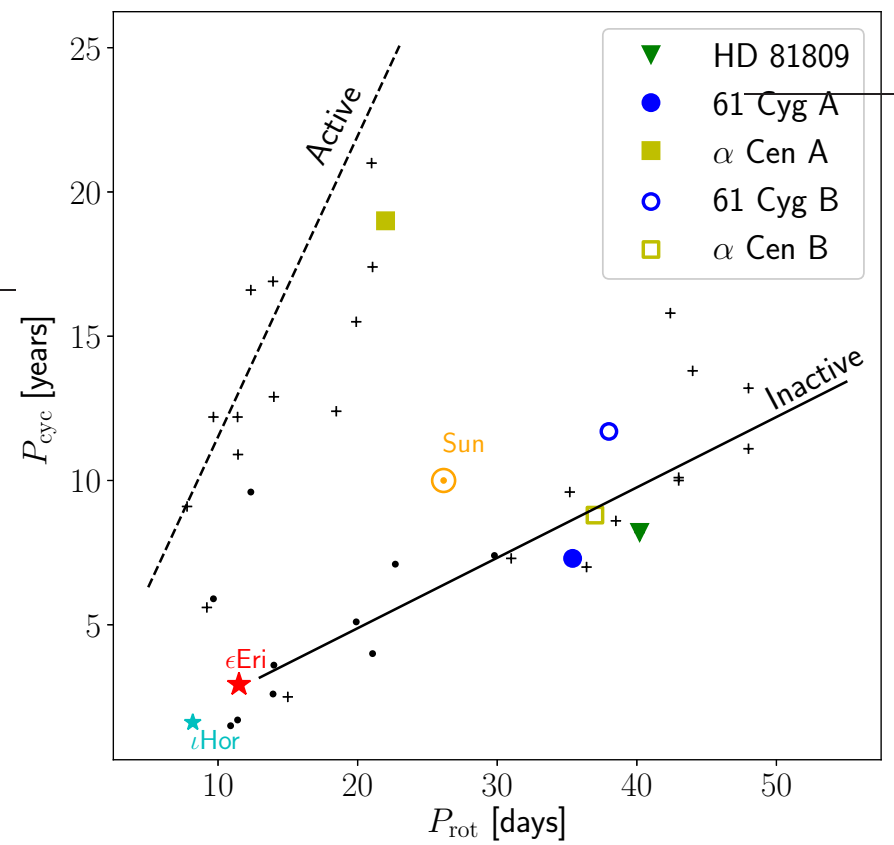

Fig. 12: Cycle period, $P_{\text {cyc }}$, as function of the rotational period, $P_{\text {rot }}$. Dotted line - active (A) branch; solid line - inactive (I) branch; black dots and crosses - original data of Böhm-Vitense (2007), updated with the new measurements available in Brandenburg et al. (2017); the black dots are secondary periods of some stars on the A branch; Colored symbols - cycles detected in X-rays (Robrade et al. 2012; Orlando et al. 2017; Sanz-Forcada et al. 2013)

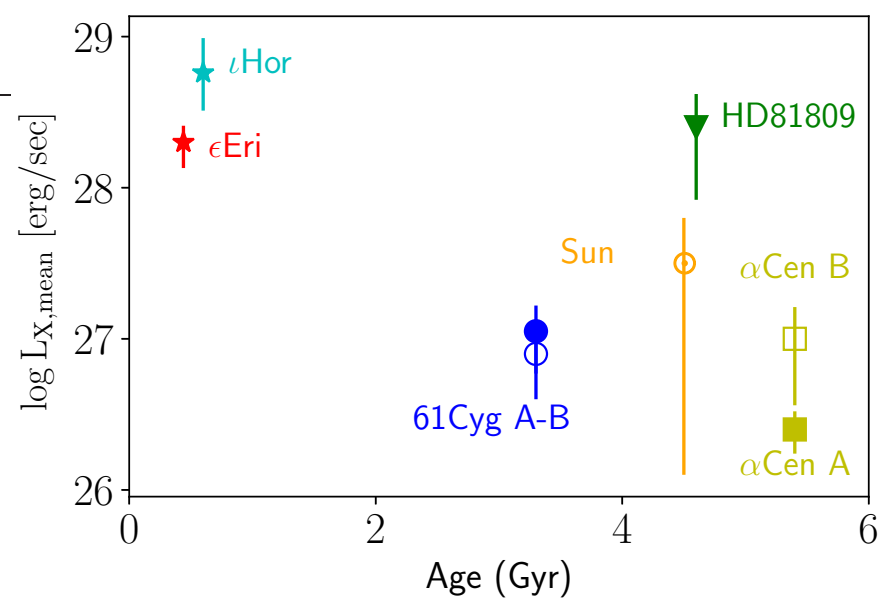

Fig. 13: The mean X-ray luminosity, $L_{X_{\text {mean }}}$, of stars with detected X-ray cycle as function of the age. $L_{X_{\text {mean }}}$ is the average of the X-ray available observations of each stars. The vertical bars are the cycle amplitudes.

ARs, COs and FLs) from a statistical assessment of the abovementioned fluctuations, as demonstrated in Fig. 7.

As a result of our detailed analysis, we found that the emission measure distributions of $\epsilon$ Eri match the solar templates only when we assume flares that are not representative of the solar average flare distribution. The best match with the observed X-ray spectra of $\epsilon$ Eri is found for flare $E M(T)$ representing solar flares during their decay. In this phase flares are cooling and, therefore, 

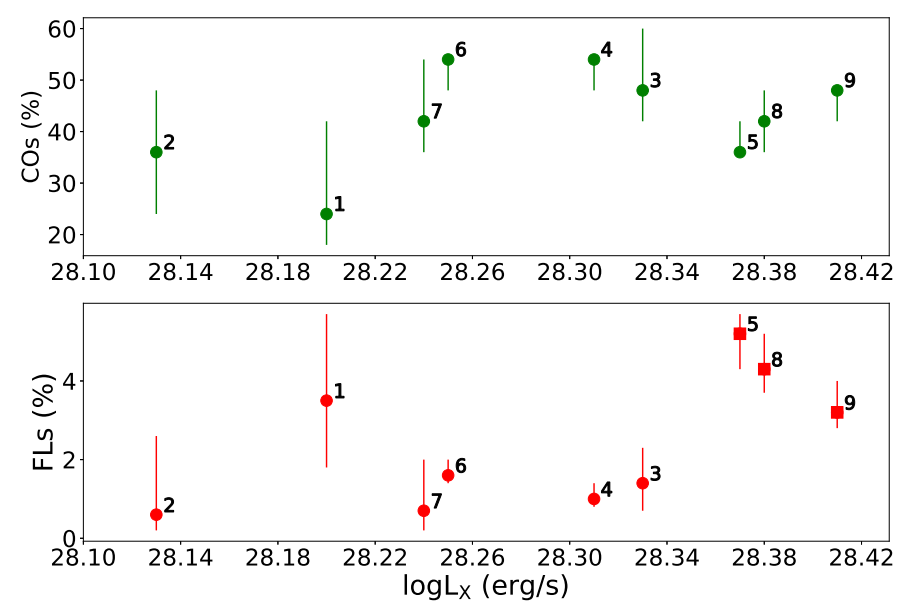

Fig. 14: Coverage fraction of COs and FLs as function of the X-ray luminosity of $\epsilon$ Eri. The top panel refers to the variation of percentage of COs that cover the surface of $\epsilon$ Eri throughout its cycle. The bottom panel shows the variation in percentage of the flaring component. The squares correspond to the observations with a flaring event in the shortterm lightcurve. The error bars are the percentile at $10 \%$ and $90 \%$ of the 1000 best-matching representations selected for each observation.

their EMD results shifted to lower temperatures with respect to the time-averaged solar flare EMD. A possible interpretation of this finding is that the actual flare distribution on $\epsilon$ Eri at any given time is dominated by flares in their late stage of evolution. Such a scenario implies that the typical duration of X-ray flares on $\epsilon$ Eri is longer than the duration of their solar counterparts. This could be due to the lower metallicity, compared to the Sun, that makes radiative losses less efficient.

In Fig. 14 we show the coverage fraction of COs and FLs throughout the cycle of $\epsilon$ Eri as function of its X-ray luminosity. The percentages of COs and FLs are the ones from our best-matching trial EMD, i.e. the one from Sect. 3.2.2, where the flares are in the decay phase and both hard and soft filters were used in the Yohkoh observations (flare EMD in green in Fig. 10) ${ }^{7}$. During the minima of the cycle (January 2003 and February 2015) the coverage of cores of active regions is $24 \%$ and $36 \%$ respectively, while in the other phases of the cycle it increases up to $54 \%$. Outside the cycle minimum the cores are thus the dominating magnetic structures in terms of spatial coverage of the corona of $\epsilon$ Eri. The maxima of the cycle require an increased percentage of flares: $\sim 3 \%$ to $\sim 5 \%$, compared to $<2 \%$ for states of lower $L_{X}$. This is in agreement with the short-term lightcurves that show flare-like variability predominately during the cycle maximum (see Sect. 2.2.2 and figures in Appendix A). Thus, the flares are superposed on the cyclic variability in the Xray waveband and therefore they have to be disentangled from the cycle variations.

In the solar cycle the flaring component observed on the Sun influences weakly the total solar corona having a marginal contribution throughout the cycle, as well as the cores of active regions that result absent in the cycle minimum and feeble in the cycle maximum (Orlando et al. 2001). Instead, for the cycle of $\epsilon$ Eri we have shown that during the minimum $44-76 \%$ of the total surface results to be covered by magnetic structures $(40 \%$

\footnotetext{
${ }^{7}$ While the test where the flare EMD is restricted to the use of the hard filter (red distribution in Fig. 10 (see Sect. 3.2.3) provides similar results, the exclusion of the soft flare component is artificial and we do not adopt this as the final EMD.
}

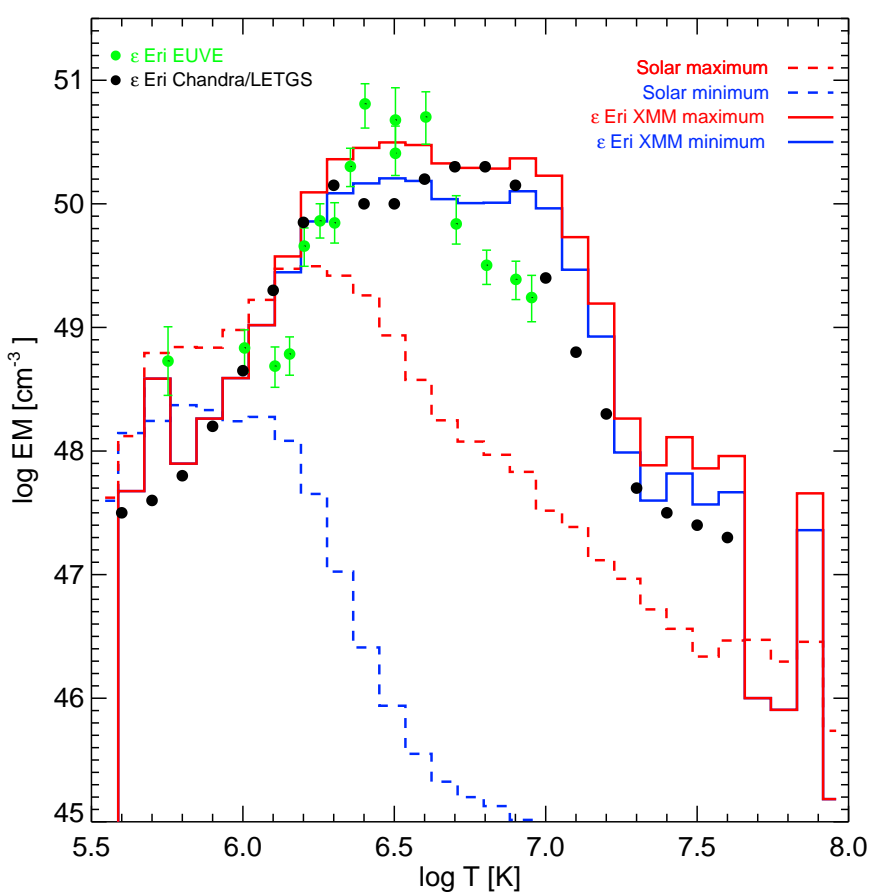

Fig. 15: EMDs of the Sun and $\epsilon$ Eri. Dashed lines: solar EMDs during the minimum in April 1996 (blue) and the maximum in December 1991 (red) of the solar cycle; solid lines: EPIC/pn EMDs of $\epsilon$ Eri during the minimum in February 2015 (blue) and maximum in July 2018 (red) of its X-ray cycle; green dots: EMD of $\epsilon$ Eri from the EUVE spectra of 1993 (Drake et al. 2000); blacks dots: EMD of $\epsilon$ Eri from Chandra/LETGS spectrum taken in March 2001 (Sanz-Forcada et al. 2004).

ARs and $24-36 \%$ COs), going up to $88 \%$ in the maximum (40\% ARs and $48 \%$ COs).

In the past Drake et al. (2000) directly compared the emission measure distribution of the solar active regions extracted from Yohkoh images with the EMD found for $\epsilon$ Eri with EUVE spectra obtained in 1993. Sanz-Forcada et al. (2003, 2004) also analyzed EMDs of $\epsilon$ Eri using EUVE and the X-ray satellite Chandra, deriving a similar shape to that of Drake et al. (2000) in the case of the EUVE data. Both these studies are based on an analysis of the high resolution spectra of $\epsilon$ Eri. In Fig. 15 we compare these EMDs (green and black dots) to the emission measure distributions of the Sun during the minimum and the maximum of the solar cycle (April 1996 and December 1991; dashed distributions) and the EMDs of $\epsilon$ Eri during the minimum and the maximum of the X-ray cycle derived by us with an entirely different method (February 2015 and August 2018; solid distributions). The EMDs found with our method are in good agreement with the X-ray EMD derived by Sanz-Forcada et al. (2004), strengthening the validity of our analysis that, even if we used low-resolution spectra, is able to reproduce past results based on high-resolution spectra. At low temperatures $\left(T \sim 10^{6}\right.$ $\mathrm{K})$, we reproduce well the emission measure of $\epsilon$ Eri presented by Drake et al. (2000) and its rise towards higher temperatures that reach a first peak at $T \sim 10^{6.5} \mathrm{~K}$. At high temperatures the X-ray emission measures show a different maximum than the EUVE data. The peaks at $\sim 10^{7} \mathrm{~K}$ in the X-ray emission measures found in our analysis are the contribution of the flaring events. The absence of this peak in the EMD obtained with $E U V E$ shows that these data have no sensitivity for the flaring component. Moreover, at the time of EUVE observations in 1993 $\epsilon$ Eri did not show the 3 -yr chromospheric cycle, i.e. the EMD 
could have been different, and thus it is reasonable to think that the current corona of $\epsilon$ Eri has more flaring events than it had 26 years ago. It can be seen from Fig. 15 that the EMDs of $\epsilon$ Eri are very different from those of the Sun throughout its activity cycle. As noticed by Drake et al. (2000), these difference may lay on the fact that active stars may be covered with more active regions than the Sun. With our method we confirmed that the high $\mathrm{X}$-ray emission from $\epsilon$ Eri can, indeed, be explained by a high surface coverage of magnetic structures.

\section{Conclusions}

We have analyzed in detail the long-term X-ray variability of $\epsilon$ Eri and we showed evidence for an X-ray cycle for the first time. With an age of $440 \mathrm{Myr}, \epsilon$ Eri, together with $\iota$ Hor, are the youngest stars with detected X-ray activity cycles.

We have applied a new method in which we describe the Xray spectra of $\epsilon$ Eri and the evolution of the X-ray cycle in terms of solar emission measure distributions considering active regions, cores of active regions and flares in the corona. We found that during the minimum of the cycle, $\sim 60 \%$ of the surface of $\epsilon$ Eri is covered by these structures, going up to 90\% throughout the X-ray cycle. We have thus found direct evidence that the high X-ray luminosity of $\epsilon$ Eri, and likely of young fast rotating stars in general, are the result of high magnetic filling factor in the corona. This result is also bolstered by the small amplitudes of the X-ray cycle of $\epsilon$ Eri, and the similarly young star $\iota$ Hor, which - in the vein of the above arguments - can be explained as a lack of additional space for enhancing the covering fraction throughout the cycle. Therefore, this suggests that an age of $\sim 400 \mathrm{Myr}$ is the youngest age for coronal X-ray cycles to set in as in even younger stars the basal surface coverage with active structures is likely to be even higher.

We found that the corona of $\epsilon$ Eri can be described in terms of solar magnetic structures only if the standard solar flare EMD is replaced by an EMD representing exclusively the decay phase of flares. We conclude that the flaring events that take place on the surface of $\epsilon$ Eri last longer than typical solar flares and we ascribe this to the low metallicity of $\epsilon$ Eri which slows down the radiative cooling in the corona.

$\epsilon$ Eri is now entering in an interesting state in which X-ray and $\mathrm{Ca}$ II cycle are not as well correlated as in the years before: while the long-term X-ray lightcurve seems to indicate an anticipation of the latest cycle maximum, the CaII $S_{\text {MWO-index }}$ variability has lately strongly decreased. Taken together with the historical Ca II observations (not considered in this work) where different cycle periods were found, it is clear that activity cycles on $\epsilon$ Eri are not a stable phenomenon. Our continued Ca II and $\mathrm{X}$-ray monitoring of this star will shed more light on this issue.

Our study shows that X-ray cycles can be present on young stars albeit with different characteristics than the solar cycle. To better validate this evidence, continuing to monitor the X-ray activity of such targets is particularly important.

Acknowledgements. MC and LD acknowledge financial support by the Bundesministerium für Wirtschaft und Energie through the Deutsches Zentrum für Luft- und Raumfahrt e.V. (DLR) under the grants FKZ 50 OR 1708 and FKZ 50 OG 1602. The research leading to these results has received also funding from the European Union's Horizon 2020 Program under the AHEAD project (grant agreement 654215). T.S.M. acknowledges the support of grant AST-1812634 from the U.S. National Science Foundation. UW acknowledges funding by DLR, project FKZ 50 OR 1701, using data obtained with the TIGRE telescope, located at La Luz observatory, Mexico. TIGRE is a collaboration of the Hamburger Sternwarte, the Universities of Hamburg, Guanajuato and Liège. JSF acknowledges support from the Spanish MICINN through grant AYA2016-79425-C3-2-

\section{References}

Arnaud, K. A. 1996, in Astronomical Society of the Pacific Conference Series, Vol. 101, Astronomical Data Analysis Software and Systems V, ed. G. H. Jacoby \& J. Barnes, 17

Baliunas, S. L., Donahue, R. A., Soon, W. H., et al. 1995, ApJ, 438, 269

Barnes, S. A. 2007, ApJ, 669, 1167

Böhm-Vitense, E. 2007, ApJ, 657, 486

Brandenburg, A., Mathur, S., \& Metcalfe, T. S. 2017, ApJ, 845, 79

Brandenburg, A., Saar, S. H., \& Turpin, C. R. 1998, ApJ, 498, L51

de la Calle, I. 2019, Users Guide to the XMM-Newton Science Analysis System Di Folco, E., Thévenin, F., Kervella, P., et al. 2004, A\&A, 426, 601

Drake, J. J., Peres, G., Orlando, S., Laming, J. M., \& Maggio, A. 2000, ApJ, 545, 1074

Duncan, D. K., Vaughan, A. H., Wilson, O. C., et al. 1991, ApJS, 76, 383

Egeland, R. 2018, ApJ, 866, 80

Favata, F., Micela, G., Orlando, S., et al. 2008, A\&A, 490, 1121

Fuhrmann, K. 2004, Astronomische Nachrichten, 325, 3

Gray, D. F. \& Baliunas, S. L. 1995, ApJ, 441, 436

Hall, J. C. \& Lockwood, G. W. 1995, ApJ, 438, 404

Hall, J. C., Lockwood, G. W., \& Skiff, B. A. 2007, AJ, 133, 862

Hatzes, A. P., Cochran, W. D., McArthur, B., et al. 2000, ApJ, 544, L145

Hempelmann, A., Robrade, J., Schmitt, J. H. M. M., et al. 2006, A\&A, 460, 261

Isaacson, H. \& Fischer, D. 2010, ApJ, 725, 875

Jeffers, S. V., Petit, P., Marsden, S. C., et al. 2014, A\&A, 569, A79

Kaastra, J. S. 1992, Internal Report, updated version 2.0 (SRON-Leiden)

Killick, R. \& Eckley, I. 2014, Journal of Statistical Software, Articles, 58, 1

Maggio, A., Flaccomio, E., Favata, F., et al. 2007, ApJ, 660, 1462

Metcalfe, T. S., Buccino, A. P., Brown, B. P., et al. 2013, ApJ, 763, L26

Metcalfe, T. S., Judge, P. G., Basu, S., et al. 2009, arXiv e-prints, arXiv:0909.5464

Mewe, R., Kaastra, J. S., \& Liedhal, D. A. 1995, Legacy, 6, 16

Mewe, R., Lemen, J. R., \& van den Oord, G. H. J. 1986, A\&AS, 65, 511

Mittag, M., Hempelmann, A., Fuhrmeister, B., Czesla, S., \& Schmitt, J. H. M. M. 2018, Astronomische Nachrichten, 339, 53

Mittag, M., Robrade, J., Schmitt, J. H. M. M., et al. 2017, A\&A, 600, A119

Mittag, M., Schröder, K.-P., Hempelmann, A., González-Pérez, J. N., \& Schmitt, J. H. M. M. 2016, A\&A, 591, A89

Olspert, N., Lehtinen, J. J., Käpylä, M. J., Pelt, J., \& Grigorievskiy, A. 2018, A\&A, 619, A6

Orlando, S., Favata, F., Micela, G., et al. 2017, A\&A, 605, A19

Orlando, S., Peres, G., \& Reale, F. 2000, ApJ, 528, 524

Orlando, S., Peres, G., \& Reale, F. 2001, ApJ, 560, 499

Orlando, S., Peres, G., \& Reale, F. 2004, A\&A, 424, 677

Peres, G., Orlando, S., Reale, F., Rosner, R., \& Hudson, H. 2000, ApJ, 528, 537

Piskunov, N. E. \& Valenti, J. A. 2002, A\&A, 385, 1095

Quillen, A. C. \& Thorndike, S. 2002, ApJ, 578, L149

Radick, R. R., Lockwood, G. W., Henry, G. W., Hall, J. C., \& Pevtsov, A. A. 2018, ApJ, 855, 75

Reale, F., Peres, G., \& Orlando, S. 2001, ApJ, 557, 906

Robrade, J., Schmitt, J. H. M. M., \& Favata, F. 2012, A\&A, 543, A84

Rutten, R. G. M. 1984, A\&A, 130, 353

Saar, S. H. \& Brandenburg, A. 1999, ApJ, 524, 295

Sanz-Forcada, J., Brickhouse, N. S., \& Dupree, A. K. 2003, ApJS, 145, 147

Sanz-Forcada, J., Favata, F., \& Micela, G. 2004, A\&A, 416, 281

Sanz-Forcada, J., Stelzer, B., Coffaro, M., Raetz, S., \& Alvarado-Gómez, J. D. 2019, A\&A, 631, A45

Sanz-Forcada, J., Stelzer, B., \& Metcalfe, T. S. 2013, A\&A, 553, L6

See, V., Jardine, M., Vidotto, A. A., et al. 2016, Monthly Notices of the Royal Astronomical Society, 462, 4442

Song, I., Caillault, J. P., Barrado y Navascués, D., Stauffer, J. R., \& Rand ich, S. 2000, ApJ, 533, L41

van Leeuwen, F. 2007, A\&A, 474, 653

Wargelin, B. J., Saar, S. H., Pojmański, G., Drake, J. J., \& Kashyap, V. L. 2016, Monthly Notices of the Royal Astronomical Society, 464, 3281

Wilson, O. C. 1968, ApJ, 153, 221

Wilson, O. C. 1978, ApJ, 226, 379

Zechmeister, M. \& Kürster, M. 2009, A\&A, 496, 577 


\section{Appendix A: EPIC/pn lightcurve}

The EPIC/pn lightcurves are shown. The time bin size chosen is set on $300 \mathrm{~s}$. The solid red lines overplotted on each lightcurve represent the segmentation found with the software $R$.
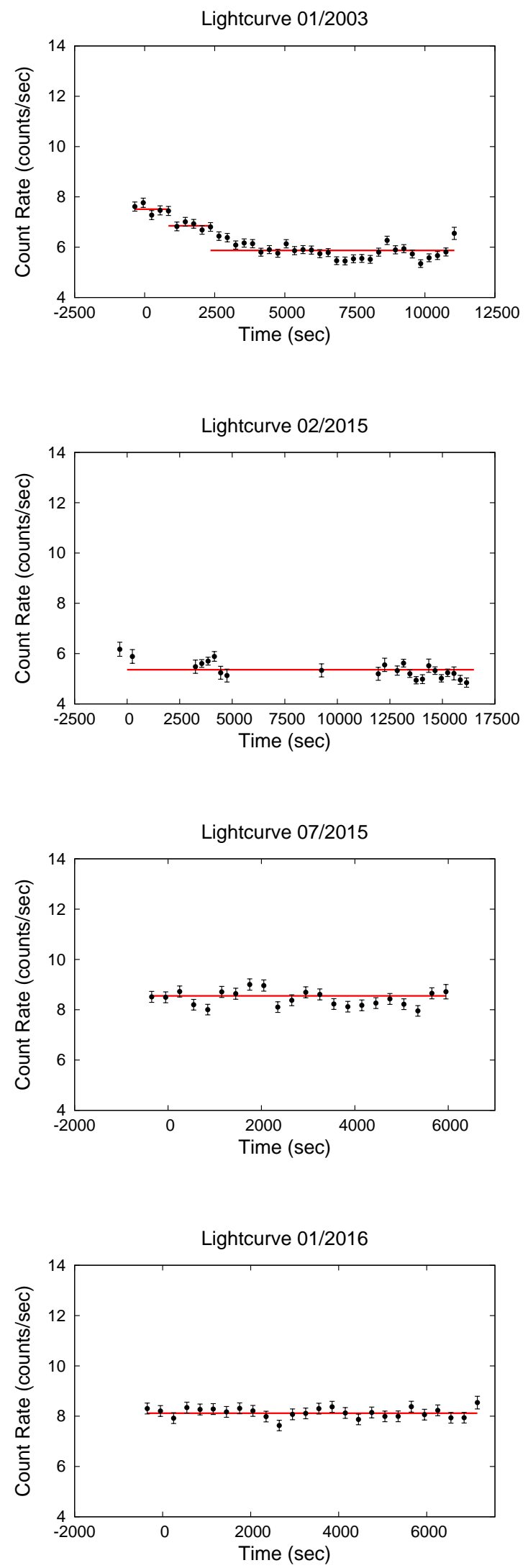

Lightcurve 07/2016

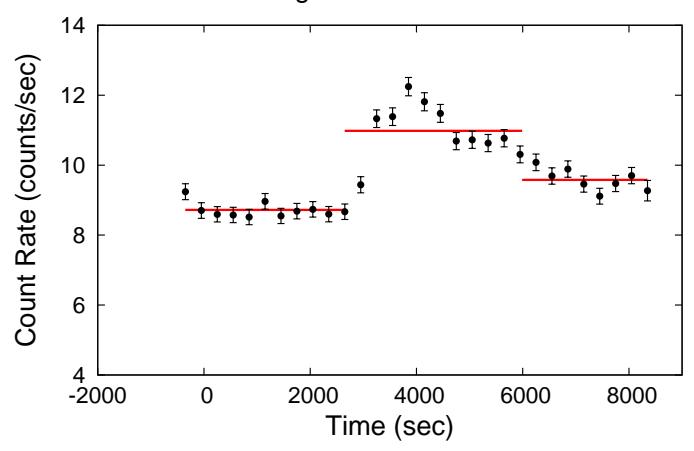

Lightcurve 01/2017

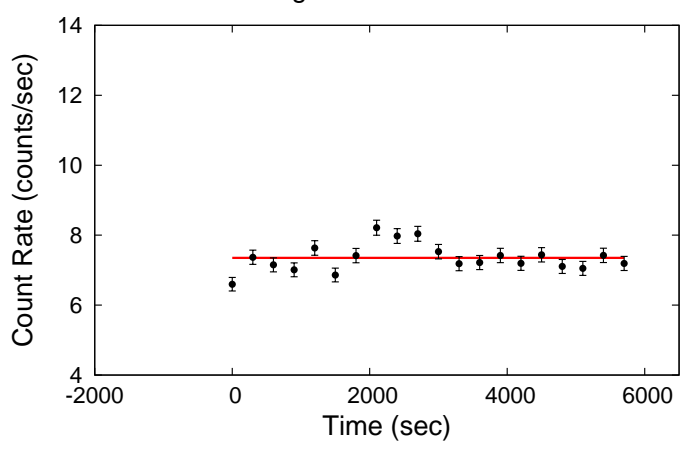

Lightcurve 08/2017

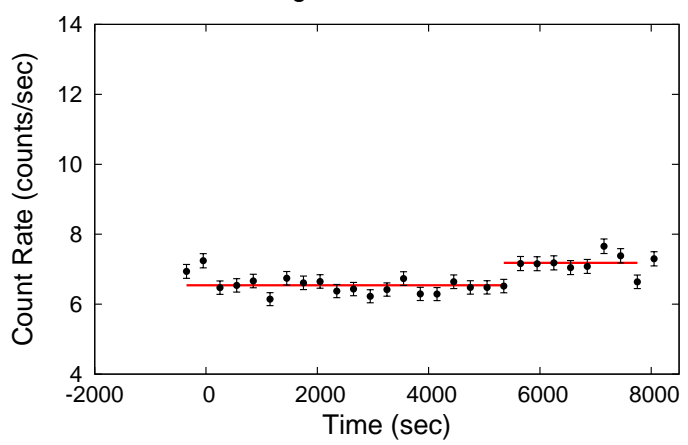

Lightcurve 01/2018

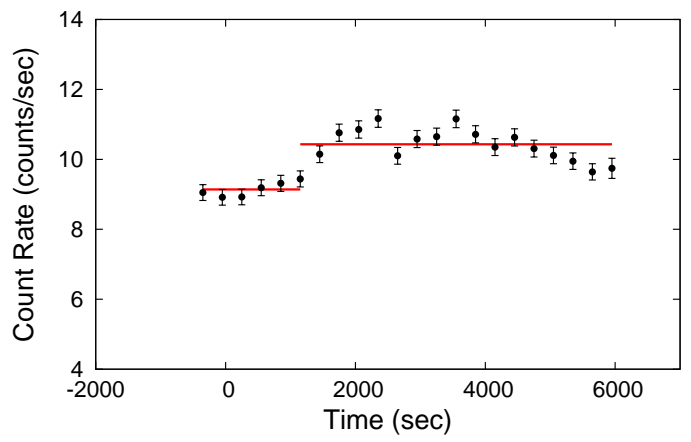




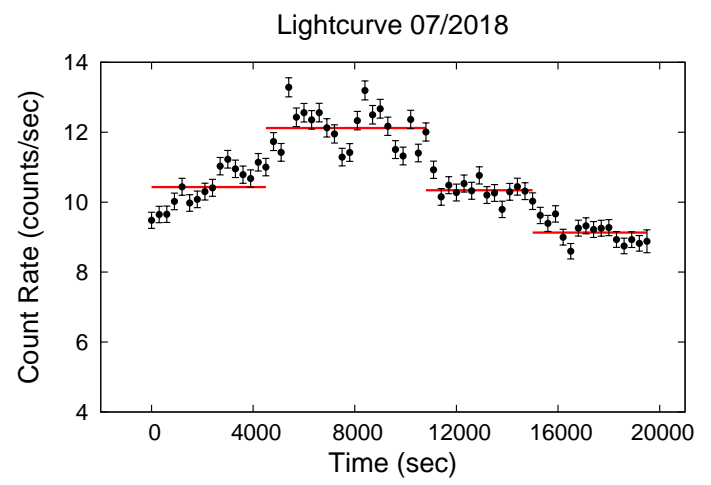




\section{Appendix B: Modified solar coronal EMD}

The EMDs selected according to the criterion given in Sect. 3.1 are shown for the three modified flare $\operatorname{EM}(\mathrm{T})$ considered in this work and discussed in Sect. 3.2. 

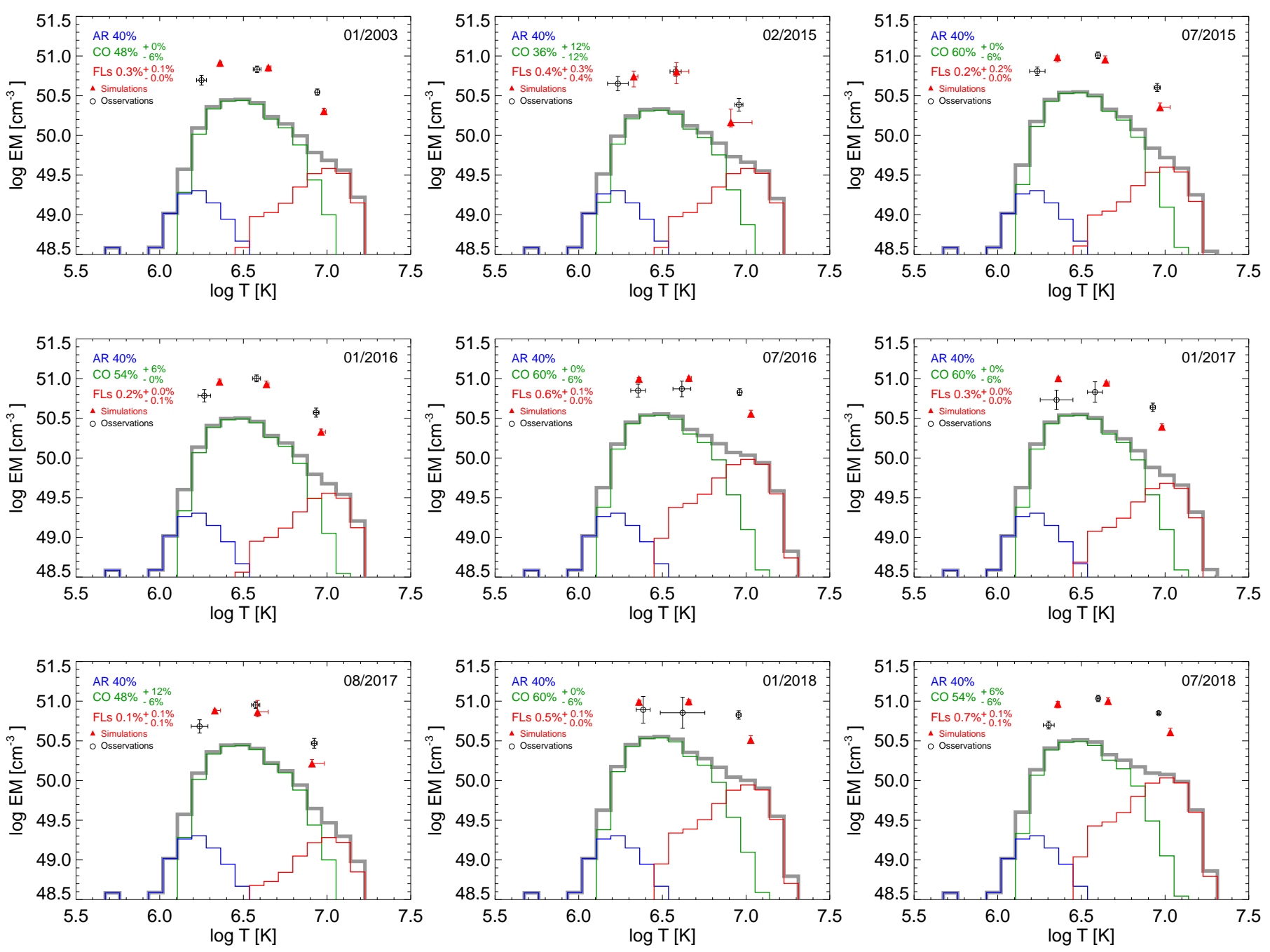

Fig. B.1: Selected best-fitting EMD for class M flares at the soft and hard energies. The coding of the plots is the same as in Fig. 8 . 

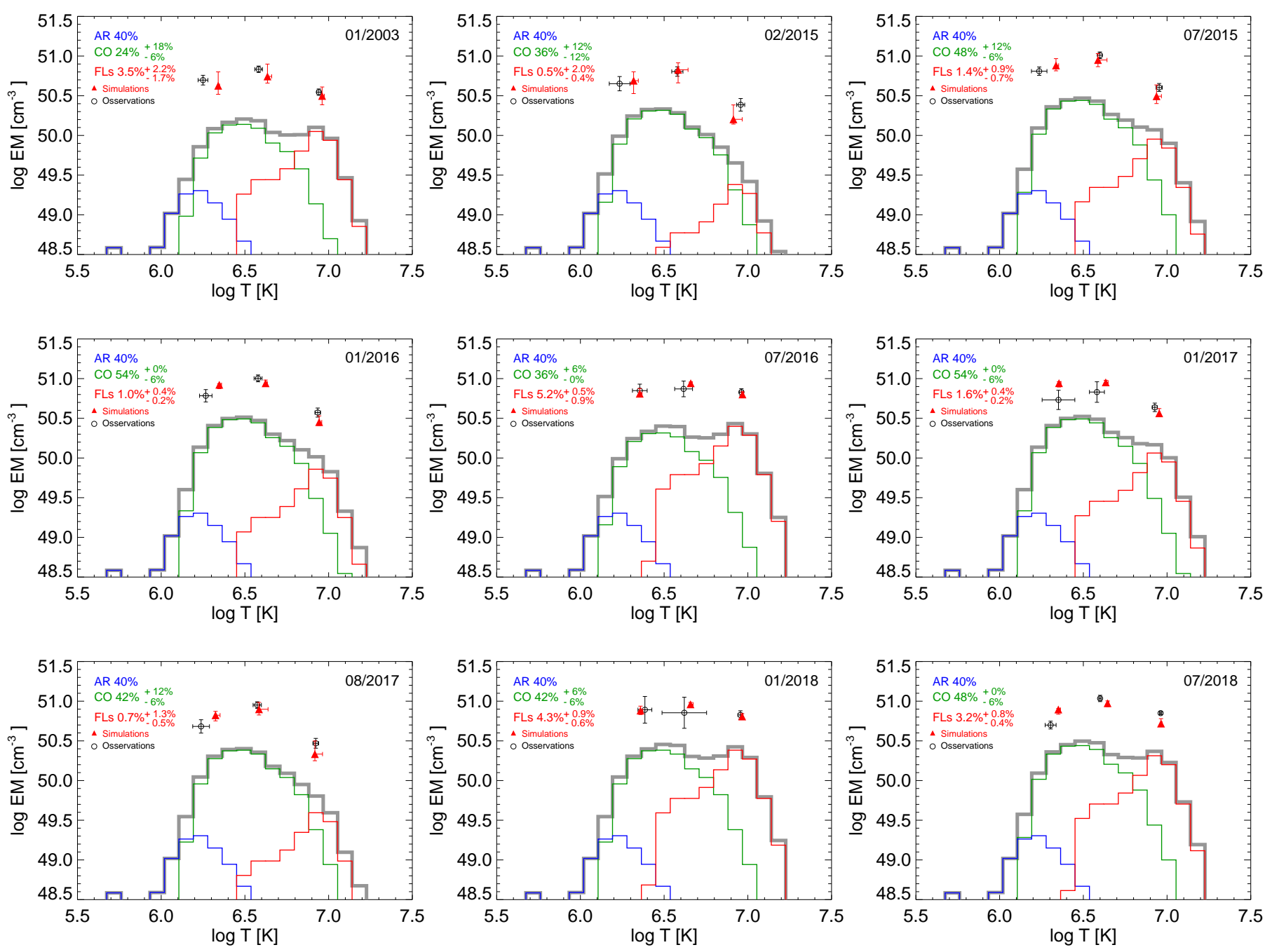

Fig. B.2: Selected best-fitting EMD for class M flares at the soft and hard energies during the decay phase. The coding of the plots is the same as in Fig. 8. 

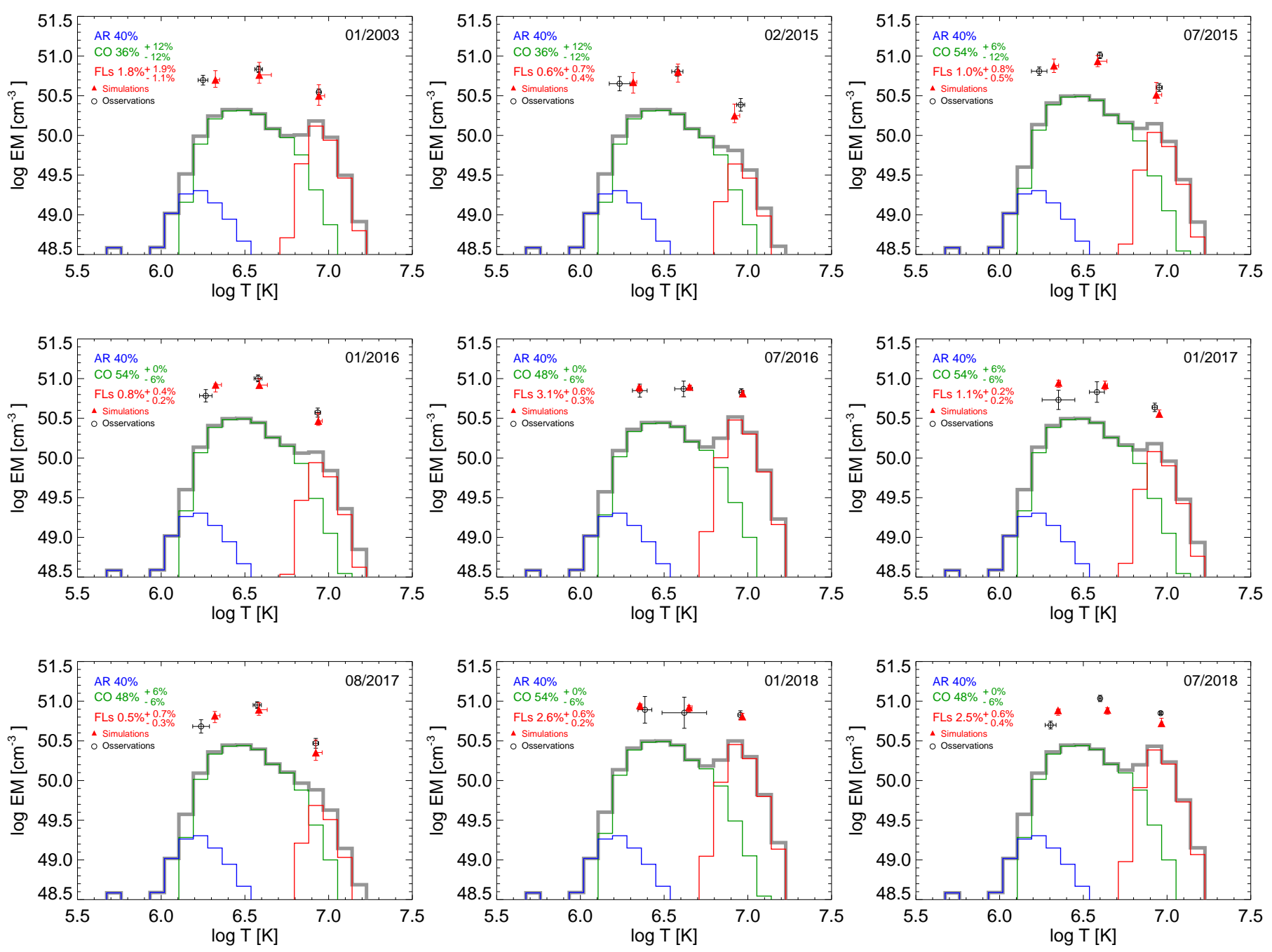

Fig. B.3: Selected best-fitting EMD for class M flares at the hard energies during the decay phase. The coding of the plots is the same as in Fig. 8 . 